\title{
Stochastic Target Games and Dynamic Programming via Regularized Viscosity Solutions*
}

\author{
Bruno Bouchard ${ }^{\dagger} \quad$ Marcel Nutz $z^{\ddagger}$
}

This version January 2015

\begin{abstract}
We study a class of stochastic target games where one player tries to find a strategy such that the state process almost-surely reaches a given target, no matter which action is chosen by the opponent. Our main result is a geometric dynamic programming principle which allows us to characterize the value function as the viscosity solution of a non-linear partial differential equation. Because abstract measurable selection arguments cannot be used in this context, the main obstacle is the construction of measurable almost-optimal strategies. We propose a novel approach where smooth supersolutions are used to define almost-optimal strategies of Markovian type, similarly as in verification arguments for classical solutions of Hamilton-Jacobi-Bellman equations. The smooth supersolutions are constructed by an extension of Krylov's method of shaken coefficients. We apply our results to a problem of option pricing under model uncertainty with different interest rates for borrowing and lending.
\end{abstract}

Keywords: Stochastic target; Stochastic differential game; Knightian uncertainty; Shaking of coefficients; Viscosity solution

AMS 2000 Subject Classification 93E20; 49L20; 91B28

\footnotetext{
*We are grateful to Erhan Bayraktar, Pierre Cardaliaguet and two anonymous referees for valuable discussions and suggestions.

${ }^{\dagger}$ CEREMADE, Université Paris Dauphine and CREST-ENSAE, bouchard@ceremade.dauphine.fr. Research supported by ANR Liquirisk and Labex ECODEC.

${ }^{\ddagger}$ Departments of Statistics and Mathematics, Columbia University, New York, mnutz@columbia.edu. Research supported by NSF Grant DMS-1208985.
} 


\section{Introduction}

We study a stochastic (semi) game where we try to find a strategy $\mathfrak{u}$ such that the controlled state process almost-surely reaches a given target at the time horizon $T$, no matter which control $\alpha$ is chosen by the adverse player. Here $\alpha$ is any predictable process with values in a given bounded set $A \subset \mathbb{R}^{d}$, whereas $\mathfrak{u}$ is a non-anticipating strategy which associates to each $\alpha$ a predictable process $\mathfrak{u}[\alpha]$ with values in a given set $U \subset \mathbb{R}^{d}$. More precisely, if $\mathcal{A}$ and $\mathcal{U}$ denote the collections of predictable processes with values in $A$ and $U$, respectively, then $\mathfrak{u}$ is a map $\mathcal{A} \rightarrow \mathcal{U}$ such that $\left.\mathfrak{u}[\alpha]\right|_{[0, s]}=\left.\mathfrak{u}\left[\alpha^{\prime}\right]\right|_{[0, s]}$ on the set $\left\{\left.\alpha\right|_{[0, s]}=\left.\alpha^{\prime}\right|_{[0, s]}\right\}$, for all $\alpha, \alpha^{\prime} \in \mathcal{A}$ and $s \leq T$. We denote by $\mathfrak{U}$ the collection of all such strategies.

Given an initial condition $(x, y) \in \mathbb{R}^{d} \times \mathbb{R}$ at time $t$ and $(\mathfrak{u}, \alpha) \in \mathfrak{U} \times \mathcal{A}$, the $(d+1)$-dimensional state process $\left(X_{t, x}^{\alpha}, Y_{t, x, y}^{\mathfrak{u}, \alpha}\right)(s), t \leq s \leq T$ is defined as the solution of the stochastic differential equation

$$
\left\{\begin{array}{l}
d X(s)=\mu_{X}\left(s, X(s), \alpha_{s}\right) d s+\sigma_{X}\left(s, X(s), \alpha_{s}\right) d W_{s} \\
d Y(s)=\mu_{Y}\left(s, X(s), Y(s), \mathfrak{u}[\alpha]_{s}, \alpha_{s}\right) d s+\sigma_{Y}\left(s, X(s), Y(s), \mathfrak{u}[\alpha]_{s}, \alpha_{s}\right) d W_{s}
\end{array}\right.
$$

where $W$ is a Brownian motion and the coefficients satisfy suitable continuity and growth conditions. Given a measurable function $g$, the value function of the stochastic target game is then given by

$$
v(t, x)=\inf \left\{y \in \mathbb{R}: \exists \mathfrak{u} \in \mathfrak{U} \text { s.t. } Y_{t, x, y}^{\mathfrak{u}, \alpha}(T) \geq g\left(X_{t, x}^{\alpha}(T)\right) \text { a.s. } \forall \alpha \in \mathcal{A}\right\} .
$$

That is, $v(t, x)$ is the smallest $y$ from which we can drive the state process into the epigraph of $g$ by using a strategy $\mathfrak{u}$ to react to the adverse player. The aim of this paper is to provide a dynamic programming principle for the stochastic target game and to characterize $v$ in terms of a Hamilton-Jacobi-Bellman equation.

In the case where $A$ is a singleton and $\mathfrak{u}$ is a control, the above is a standard stochastic target problem in the terminology of [25, 26]. In [25], it is shown that the value function of this target problem satisfies a geometric dynamic programming principle (GDP) and, consequently, is a discontinuous viscosity solution of an associated Hamilton-Jacobi-Bellman equation. The GDP consists of two parts, called GDP1 and GDP2. Roughly, given a family $\left\{\theta^{\mathfrak{u}}, \mathfrak{u} \in \mathfrak{U}\right\}$ of stopping times with values in $[t, T]$, GDP1 states that

$$
\text { if } y>v(t, x) \text {, then there exists } \mathfrak{u} \in \mathfrak{U} \text { such hat } Y_{t, y}^{\mathfrak{u}, a}\left(\theta^{\mathfrak{u}}\right) \geq v\left(\theta^{\mathfrak{u}}, X_{t, x}^{a}\left(\theta^{\mathfrak{u}}\right)\right) \text {, }
$$

and conversely, GDP2 states that

if there exists $\mathfrak{u} \in \mathfrak{U}$ such that $Y_{t, y}^{\mathfrak{u}, a}\left(\theta^{\mathfrak{u}}\right)>v\left(\theta^{\mathfrak{u}}, X_{t, x}^{a}\left(\theta^{\mathfrak{u}}\right)\right)$, then $y \geq v(t, x)$.

The line of argument for GDP1 can be reproduced in the context of games. However, all previous proofs of GDP2 crucially rely on the construction of almost optimal controls through measurable selections. It is well known that in the context of games, when $\mathfrak{u}$ is a strategy as defined above, the possibility of using such a selection is a completely open problem. The main difficulties come from the lack of separability in the space $\mathfrak{U}$ of strategies and the irregular dependence on the adverse control $\alpha$.

For zero-sum differential games in standard form, see e.g. [12] and [8], there are by now several workarounds for this problem; they rely on approximations and 
exploit the continuity properties of the cost functions. We also refer to [23] and 24 for recent developments in different directions, in a setting where both players use controls. In [6], stochastic target games were analyzed when the target is of controlled-loss type; that is, of the form $\mathbb{E}\left[\ell\left(X_{t, x, y}^{\mathfrak{u}, \alpha}(T), Y_{t, x, y}^{\mathfrak{u}, \alpha}(T)\right)\right] \geq m$, where $m$ is given and the loss function $\ell$ has certain continuity properties. Again, the regularity of $\ell$ was exploited to circumvent the problem of measurable selection. By contrast, the almost-sure target of the game under consideration is highly discontinuous, which has prevented us from arguing similarly as in the mentioned works.

In this paper, we follow a completely different and novel idea. As a starting point, recall that in the context of standard control problems with a smooth value function, we can sometimes use a verification argument. Here, an optimal control of Markovian (i.e. feedback) type is defined explicitly in terms of the derivatives of the value function. It plays the role of the almost-optimal controls mentioned above and renders measurable selection arguments unnecessary. Of course, this procedure requires a smooth value function, which cannot be expected in our context. However, it will turn out that a smooth supersolution with specific properties can be used in a similar spirit. The outline of our argument runs as follows.

(a) Show that the value function $v$ satisfies a version of GDP1 above (Theorem 2.3).

(b) Deduce from GDP1 that $v$ is a viscosity supersolution of the associated Hamilton-Jacobi-Bellman equation (Theorem 2.5).

(c) Regularize $v$ to find a smooth supersolution $w$ which is close to $v$ is a specific sense (Lemma 2.6).

(d) Using $w$, construct a strategy of Markovian type that matches the criterion in (1.1) when starting slightly above $v(t, x)$ and use this strategy to prove a version of GDP2 (Theorem 2.7).

(e) Deduce from GDP2 that $v$ is also a viscosity subsolution of the HamiltonJacobi-Bellman equation (Theorem 2.8).

The arguments for (a) and (b) are along the lines of [25], while at least part of the proof of (e) follows [6]. The construction of the smooth supersolution in (c) is based on Krylov's method of shaking the coefficients from [15, Theorem 2.1] (see also [2]), which we extend here to semi-linear equations by considering value functions of controlled forward-backward stochastic differential equations (Theorem 3.3). We mention that this method imposes a concavity condition on one of the operators. On the other hand, to the best of our knowledge, our result is the first proof of dynamic programming for stochastic target games with almost-sure target 1

Our results can be compared to the second order backward stochastic differential equations of [28, where the authors use quasi-sure analysis in the "weak formulation". Their setting is more general in the sense that path-dependence is allowed and concavity is not needed. On the other hand, we allow nonlinear dynamics for $X$, while their setting corresponds to the case where $\sigma_{X}(\cdot, a)=a$ and $\mu_{X}=0$.

\footnotetext{
${ }^{1}$ Note added in proof: In the follow-up work [4, a similar target game problem is treated by a stochastic Perron's method which does not require a dynamic programming principle.
} 
We apply our results to a problem from mathematical finance where an option is to be super-hedged under model uncertainty. The model uncertainty is addressed in a worst-case fashion, so that it can be modeled by an adverse player as above. More precisely, the drift and volatility of the underlying of the option as well as the two interest rates for borrowing and lending depend on the adverse control $\alpha$. Various incarnations of the super-hedging problem have been considered in the recent literature; see [1, 7, 11, 16, 22, 27, 29] and the references therein. The now standard approach is to use the weak formulation where the uncertainty is modeled by a non-dominated set $\mathcal{P}$ of possible laws on path space. The super-hedging property is then required to hold almost-surely under each element of $\mathcal{P}$ and the study involves the difficulty of dealing with a non-dominated set of probabilities (quasi-sure analysis). We adopt here a different point of view, where uncertainty is modeled as a game under a single probability measure; namely, the adverse player ("nature") chooses drift and volatility while we can react by using a suitable non-anticipating strategy and the superhedging-property is required to hold almostsurely for any control of the adverse player. Our formulation is thus in the spirit of [30] and 31] where problems of portfolio management are phrased as stochastic differential games of standard form in the framework of 12 .

The remainder of this paper is organized as follows. In Section 2, we formulate the stochastic target game in detail and provide the geometric dynamic programming principle together with the Hamilton-Jacobi-Bellman equation for the value function $v$. The key part of the dynamic programming principle, GDP2, is based on the regularization result, which is developed in Section 3 in a slightly more general setting. In Section 4, we exemplify our results by the application to super-hedging under model uncertainty.

\section{Geometric dynamic programming for stochastic target games}

In this section, we first detail our problem formulation. In the second subsection, we provide the first part of the geometric dynamic programming principle, GDP1, and infer the supersolution property of the value function. In the third subsection, we prove the difficult part, GDP2, together with the subsolution property.

\subsection{Problem formulation}

Fix a time horizon $T>0$, let $\Omega$ be the space of continuous functions $\omega:[0, T] \rightarrow \mathbb{R}^{d}$ and let $\mathbb{P}$ be the Wiener measure on $\Omega$. Moreover, let $W$ be the canonical process on $\Omega$, defined by $W_{t}(\omega)=\omega_{t}$. We denote by $\mathbb{F}=\left(\mathcal{F}_{s}\right)_{0 \leq s \leq T}$ the augmented filtration generated by $W$. Furthermore, for $0 \leq t \leq T$, we denote by $\mathbb{F}^{t}=\left(\mathcal{F}_{s}^{t}\right)_{0 \leq s \leq T}$ the augmented filtration generated by $\left(W_{s}-W_{t}\right)_{s \geq t}$; by convention, $\mathcal{F}_{s}^{t}$ is trivial for $s \leq t$. We denote by $\mathcal{U}^{t}$ (resp. $\mathcal{A}^{t}$ ) the collection of all $\mathbb{F}^{t}$-predictable processes in $L^{p}(\mathbb{P} \otimes d t)$ with values in a given Borel subset $U$ (resp. bounded closed subset $A$ ) of $\mathbb{R}^{d}$, where $p \geq 2$ is fixed throughout. Finally, let

$$
\begin{gathered}
\mathcal{D}:=[0, T] \times \mathbb{R}^{d}, \quad \mathcal{D}_{<T}:=[0, T) \times \mathbb{R}^{d}, \quad \mathcal{D}_{T}:=\{T\} \times \mathbb{R}^{d} . \\
\text { Given }(t, x, y) \in \mathcal{D} \times \mathbb{R} \text { and }(\nu, \alpha) \in \mathcal{U}^{t} \times \mathcal{A}^{t}, \text { we let }\left(X_{t, x}^{\alpha}, Y_{t, x, y}^{\nu, \alpha}\right)(s), t \leq s \leq T
\end{gathered}
$$


be the unique strong solution of

$$
\left\{\begin{array}{l}
d X(s)=\mu_{X}\left(s, X(s), \alpha_{s}\right) d s+\sigma_{X}\left(s, X(s), \alpha_{s}\right) d W_{s} \\
d Y(s)=\mu_{Y}\left(s, X(s), Y(s), \nu_{s}, \alpha_{s}\right) d s+\sigma_{Y}\left(s, X(s), Y(s), \nu_{s}, \alpha_{s}\right) d W_{s}
\end{array}\right.
$$

with initial data $(X(t), Y(t))=(x, y)$. The coefficients $\mu_{X}, \mu_{Y}, \sigma_{Y}$ and $\sigma_{X}$ are supposed to be continuous in all variables, uniformly in the last one, and take values in $\mathbb{R}^{d}, \mathbb{R}, \mathbb{R}^{d}$ and $\mathbb{M}^{d}:=\mathbb{R}^{d \times d}$, respectively. (Elements of $\mathbb{R}^{d}$ as viewed as column vectors). We assume throughout that there exists $K>0$ such that

$$
\begin{array}{r}
\left|\mu_{X}(\cdot, x, \cdot)-\mu_{X}\left(\cdot, x^{\prime}, \cdot\right)\right|+\left|\sigma_{X}(\cdot, x, \cdot)-\sigma_{X}\left(\cdot, x^{\prime}, \cdot\right)\right| \leq K\left|x-x^{\prime}\right|, \\
\left|\mu_{X}(\cdot, x, \cdot)\right|+\left|\sigma_{X}(\cdot, x, \cdot)\right| \leq K \\
\left|\mu_{Y}(\cdot, y, \cdot)-\mu_{Y}\left(\cdot, y^{\prime}, \cdot\right)\right|+\left|\sigma_{Y}(\cdot, y, \cdot)-\sigma_{Y}\left(\cdot, y^{\prime}, \cdot\right)\right| \leq K\left|y-y^{\prime}\right|, \\
\left|\mu_{Y}(\cdot, y, u, \cdot)\right|+\left|\sigma_{Y}(\cdot, y, u, \cdot)\right| \leq K(1+|u|+|y|)
\end{array}
$$

for all $(x, y),\left(x^{\prime}, y^{\prime}\right) \in \mathbb{R}^{d} \times \mathbb{R}$ and $u \in U$. In particular, this ensures that the SDE (2.1) is well-posed. Moreover, we can note that the solution is in fact adapted not only to $\mathbb{F}$ but also to $\mathbb{F}^{t}$.

Remark 2.1. For simplicity, we consider the above dynamics for any initial point $x \in \mathbb{R}^{d}$. The case where $X_{t, x}^{\alpha}$ is restricted to an open domain $\mathcal{O} \subset \mathbb{R}^{d}$ will be discussed in Remark 3.4 below.

For the derivation of the viscosity supersolution property, we shall also impose a condition on the growth of $\mu_{Y}$ relative to $\sigma_{Y}$ :

$$
\sup _{u \in U} \frac{\left|\mu_{Y}(\cdot, u, \cdot)\right|}{1+\left|\sigma_{Y}(\cdot, u, \cdot)\right|} \text { is locally bounded. }
$$

Let $t \leq T$. We say that a map $\mathfrak{u}: \mathcal{A}^{t} \rightarrow \mathcal{U}^{t}, \alpha \mapsto \mathfrak{u}[\alpha]$ is a $t$-admissible strategy if it is non-anticipating in the sense that

$$
\left\{\omega \in \Omega:\left.\alpha(\omega)\right|_{[t, s]}=\left.\alpha^{\prime}(\omega)\right|_{[t, s]}\right\} \subset\left\{\omega \in \Omega:\left.\mathfrak{u}[\alpha](\omega)\right|_{[t, s]}=\left.\mathfrak{u}\left[\alpha^{\prime}\right](\omega)\right|_{[t, s]}\right\} \text { a.s. }
$$

for all $s \in[t, T]$ and $\alpha, \alpha^{\prime} \in \mathcal{A}^{t}$, where $\left.\right|_{[t, s]}$ indicates the restriction to the interval $[t, s]$. We denote by $\mathfrak{U}^{t}$ the collection of all $t$-admissible strategies; moreover, we write $Y_{t, x, y}^{\mathfrak{u}, \alpha}$ for $Y_{t, x, y}^{\mathfrak{u}[\alpha], \alpha}$. Finally, let $g: \mathbb{R}^{d} \rightarrow \mathbb{R}$ be a measurable function; then we can introduce the value function of our stochastic target game,

$$
v(t, x):=\inf \left\{y \in \mathbb{R}: \exists \mathfrak{u} \in \mathfrak{U}^{t} \text { s.t. } Y_{t, x, y}^{\mathfrak{u}, \alpha}(T) \geq g\left(X_{t, x}^{\alpha}(T)\right) \text { a.s. } \forall \alpha \in \mathcal{A}^{t}\right\}
$$

for $(t, x) \in \mathcal{D}$. We shall assume throughout that

$g$ is bounded and Lipschitz continuous on $\mathbb{R}^{d}$, and $v$ is bounded on $\mathcal{D}$.

Remark 2.2. The condition that $v$ is bounded has to be checked on a case-by-case basis. One typical example in which $v^{+}$is bounded is when there exists $u$ such that $\sigma_{Y}(\cdot, u, \cdot)=0$. Then, the condition (2.2) on $\mu_{Y}$ implies that $v^{+}$is bounded by $e^{K T}\left(K T+\sup _{\mathbb{R}^{d}} g\right)$. A simple situation in which $v^{-}$is bounded is when $\sigma_{Y}^{-1} \mu_{Y}$ is bounded. Then, an obvious change of measure argument allows to turn $Y_{t, x, y}^{\mathfrak{u}, \alpha}$ into a martingale, for $(\mathfrak{u}, \alpha)$ given, which implies that $v \geq \inf _{\mathbb{R}^{d}} g$. See also Section 4 , 


\subsection{First part of the dynamic programming principle and supersolution property}

We first provide one side of the geometric dynamic programming principle, GDP1, for the value function $v$ of (2.5). We denote by $v_{*}$ the lower-semicontinuous envelope of $v$ on $\mathcal{D}$.

Theorem 2.3 (GDP1). Let $(t, x, y) \in \mathcal{D} \times \mathbb{R}$ and let $\left\{\theta^{\mathfrak{u}, \alpha},(\mathfrak{u}, \alpha) \in \mathfrak{U}^{t} \times \mathcal{A}^{t}\right\}$ be a family of $\mathbb{F}^{t}$-stopping times with values in $[t, T]$. Assume that $y>v(t, x)$. Then, there exists $\mathfrak{u} \in \mathfrak{U}^{t}$ such that

$$
Y_{t, x, y}^{\mathfrak{u}, \alpha}\left(\theta^{\mathfrak{u}, \alpha}\right) \geq v_{*}\left(\theta^{\mathfrak{u}, \alpha}, X_{t, x}^{\alpha}\left(\theta^{\mathfrak{u}, \alpha}\right)\right) \text { a.s. } \forall \alpha \in \mathcal{A}^{t} .
$$

Proof. The ingredients of the proof are essentially known, so we confine ourselves to a sketch. As $y>v(t, x)$, the definition of $v$ shows that there exists $\mathfrak{u} \in \mathfrak{U}^{t}$ satisfying

$$
Y_{t, x, y}^{\mathfrak{u}, \alpha}(T) \geq g\left(X_{t, x}^{\alpha}(T)\right) \text { a.s. } \forall \alpha \in \mathcal{A}^{t} \text {. }
$$

Step 1. We first consider the case where $\theta^{\mathfrak{u}, \alpha} \equiv s \in[t, T]$ is a deterministic time independent of $\mathfrak{u}, \alpha$. To be able to write processes as functionals of the canonical process, we pass to the raw filtration. More precisely, $\overline{\mathbb{F}}^{t}$ denotes the raw filtration generated by $\left(W_{s}-W_{t}\right)_{t \leq s \leq T}$, extended trivially to $[0, T]$. By [10, Appendix I, Lemma 7], we can find for each $\alpha \in \mathcal{A}^{t}$ an $\overline{\mathbb{F}}^{t}$-predictable process $\overline{\mathfrak{u}}[\alpha]$ which is indistinguishable from $\mathfrak{u}[\alpha]$. The map $\alpha \in \mathcal{A}^{t} \mapsto \overline{\mathfrak{u}}[\alpha]$ still satisfies the non-anticipativity condition (2.4) and therefore defines an element of $\mathfrak{U}^{t}$. Moreover, (2.7) still holds if we replace $\mathfrak{u}$ by $\overline{\mathfrak{u}}$ :

$$
Y_{t, x, y}^{\overline{\mathfrak{u}}, \alpha}(T) \geq g\left(X_{t, x}^{\alpha}(T)\right) \text { a.s. } \forall \alpha \in \mathcal{A}^{t} .
$$

We claim that it suffices to show that

$$
Y_{t, x, y}^{\mathfrak{u}, \bar{\alpha}}(s) \geq v_{*}\left(s, X_{t, x}^{\bar{\alpha}}(s)\right) \text { a.s. for all } \bar{\alpha} \in \overline{\mathcal{A}}^{t},
$$

where $\overline{\mathcal{A}}^{t}$ is the set of all $\overline{\mathbb{F}}^{t}$-predictable processes with values in $A$. Indeed, if $\alpha \in \mathcal{A}^{t}$, then by [10, Appendix I, Lemma 7] we can find $\bar{\alpha} \in \overline{\mathcal{A}}^{t}$ such that $\alpha$ and $\bar{\alpha}$ are indistinguishable. In view of the non-anticipativity condition (2.4), $\mathfrak{u}[\alpha]$ and $\mathfrak{u}[\bar{\alpha}]$ are also indistinguishable, and then (2.9) implies that the same inequality holds for $\alpha$; that is, (2.9) extends from $\overline{\mathcal{A}}^{t}$ to $\mathcal{A}^{t}$.

To prove (2.9), fix $\bar{\alpha} \in \overline{\mathcal{A}}^{t}$. For given $\omega \in \Omega$, we define

$$
\overline{\mathfrak{u}}_{\omega}:(\tilde{\omega}, \tilde{\alpha}) \in \Omega \times \mathcal{A}^{s} \mapsto \overline{\mathfrak{u}}\left[\bar{\alpha}(\omega) \oplus_{s} \tilde{\alpha}\right]\left(\omega \oplus_{s}\left(\tilde{\omega}-\tilde{\omega}_{s}+\omega_{s}\right)\right),
$$

where we use the notation

$$
\gamma \oplus_{s} \gamma^{\prime}:=\gamma \mathbf{1}_{[0, s]}+\mathbf{1}_{(s, T]} \gamma^{\prime}
$$

We observe that $\overline{\mathfrak{u}}_{\omega} \in \mathfrak{U}^{s}$. Using (2.8) and the flow property of the SDE (2.1), we can find a nullset $N$ (depending on $\bar{\alpha}$ ) such that for all $\omega \notin N$ and all $\tilde{\alpha} \in \mathcal{A}^{s}$,

$$
Y_{s, x^{\prime}(\omega), y^{\prime}(\omega)}^{\overline{\mathfrak{u}}_{\omega}, \tilde{\alpha}}(T) \geq g\left(X_{s, x^{\prime}(\omega)}^{\tilde{\alpha}}(T)\right) \text { a.s. },
$$


where $x^{\prime}(\omega):=X_{t, x}^{\bar{\alpha}}(s)(\omega)$ and $y^{\prime}(\omega):=Y_{t, x, y}^{\bar{u}, \bar{\alpha}}(s)(\omega)$. By the definition of $v$, this means that $y^{\prime}(\omega) \geq v\left(s, x^{\prime}(\omega)\right)$ or

$$
Y_{t, x, y}^{\bar{u}, \bar{\alpha}}(s)(\omega) \geq v\left(s, X_{t, x}^{\bar{\alpha}}(s)(\omega)\right) \forall \omega \notin N .
$$

Since $Y_{t, x, y}^{\bar{u}, \bar{\alpha}}(s)=Y_{t, x, y}^{\mathfrak{u}, \bar{\alpha}}(s)$ a.s. and $v \geq v_{*}$, this shows that (2.9) holds.

Step 2. To deduce the case of a general family $\left\{\theta^{\mathfrak{u}, \alpha},(\mathfrak{u}, \alpha) \in \mathfrak{U}^{t} \times \mathcal{A}^{t}\right\}$ from Step 1 , we approximate each $\theta^{\mathfrak{u}, \alpha}$ from the right by stopping times with finitely many values and use the lower-semicontinuity of $v_{*}$ and the right-continuity of the paths of the state process. See e.g. [6, Section 2.3, Step 4] for a very similar argument.

We shall prove the supersolution property under the two subsequent conditions. A more general framework could be considered here (see e.g. [5] or [6]), but we shall anyway need these conditions for the second part of the dynamic programming principle below. Given $(t, x, y, z, a) \in \mathcal{D} \times \mathbb{R} \times \mathbb{R}^{d} \times A$, define the set

$$
N(t, x, y, z, a):=\left\{u \in U: \sigma_{Y}(t, x, y, u, a)=z\right\} .
$$

The first condition is that $u \mapsto \sigma_{Y}(t, x, y, u, a)$ is invertible, and more precisely:

Assumption (A). There exists a measurable map $\hat{u}: \mathcal{D} \times \mathbb{R} \times \mathbb{R}^{d} \times A \rightarrow U$ such that $N=\{\hat{u}\}$. Moreover, the map $\hat{u}(\cdot, a)$ is continuous for each $a \in A$.

The second assumption is for the boundary condition at time $T$.

Assumption (B). Fix $a \in A$ and $(x, y) \in \mathbb{R}^{d} \times \mathbb{R}$. If there exist a sequence $\left(t_{n}, x_{n}, y_{n}\right) \in \mathcal{D}_{<T} \times \mathbb{R}$ such that $\left(t_{n}, x_{n}, y_{n}\right) \rightarrow(T, x, y)$ and a sequence $\mathfrak{u}_{n} \in \mathfrak{U}^{t_{n}}$ such that $Y_{t_{n}, x_{n}, y_{n}}^{\mathfrak{u}_{n}, a}(T) \geq g\left(X_{t_{n}, x_{n}}^{a}(T)\right)$ a.s. for all $n \geq 1$, then $y \geq g(x)$.

Remark 2.4. It follows from (2.2) and (2.6) that $g\left(X_{t_{n}, x_{n}}^{a}(T)\right) \rightarrow g(x)$ a.s. for $n \rightarrow \infty$. If $U$ is bounded, then similarly $Y_{t_{n}, x_{n}, y_{n}}^{\mathfrak{u}_{n}, a}(T) \rightarrow y$ and we infer that Assumption (B) holds. In the applications we have in mind, the assumption is satisfied even when $U$ is unbounded; see the proof of Corollary 4.1 below.

To state the supersolution property, let us define for $(t, x, y, q, p, M) \in \mathcal{D} \times \mathbb{R} \times$ $\mathbb{R} \times \mathbb{R}^{d} \times \mathbb{M}^{d}$ the operators

$$
\begin{aligned}
L^{a}(t, x, y, q, p, M):= & \mu_{Y}^{\hat{u}}\left(t, x, y, \sigma_{X}(t, x, a) p, a\right)-q-\mu_{X}(t, x, a)^{\top} p \\
& -\frac{1}{2}\left[\sigma_{X} \sigma_{X}^{\top}(t, x, a) M\right]
\end{aligned}
$$

and

$$
L:=\min _{a \in A} L^{a}
$$

where

$$
\mu_{Y}^{\hat{u}}(t, x, y, z, a):=\mu_{Y}(t, x, y, \hat{u}(t, x, y, z, a), a), z \in \mathbb{R}^{d} .
$$

Theorem 2.5. Let Assumptions $(\mathbf{A})$ and $(\mathbf{B})$ hold. Then, the function $v_{*}$ is a bounded viscosity supersolution of

$$
\begin{array}{rlll}
L\left(\cdot, \varphi, \partial_{t} \varphi, D \varphi, D^{2} \varphi\right)=0 & \text { on } & \mathcal{D}_{<T} \\
\varphi-g=0 & \text { on } & \mathcal{D}_{T} .
\end{array}
$$


Proof. Now that GDP1 has already been established, the argument is similar to [5. Section 5.1] and [6. Theorem 3.4, Step 1]. We sketch the proof for completeness.

Step 1. We start with the boundary condition, which is in fact an immediate consequence of Assumption (B). Let $\left(t_{n}, x_{n}\right) \rightarrow(T, x)$ be such that $v_{*}\left(t_{n}, x_{n}\right) \rightarrow$ $v_{*}(T, x)$, for some $x \in \mathbb{R}^{d}$. Set $y_{n}:=v\left(t_{n}, x_{n}\right)+n^{-1}$. Then, we can find $\mathfrak{u}_{n} \in \mathfrak{U}^{t_{n}}$ such that $Y_{t_{n}, x_{n}, y_{n}}^{\mathfrak{u}_{n}, a}(T) \geq g\left(X_{t_{n}, x_{n}}^{a}(T)\right)$ a.s. for all $a \in A$. Sending $n \rightarrow \infty$ and using Assumption (B) yields $v_{*}(T, x) \geq g(x)$ as desired.

Step 2. We now prove that $v_{*}$ is a viscosity supersolution of (2.10) on $\mathcal{D}_{<T}$. Let $\varphi$ be a smooth function and let $\left(t_{o}, x_{o}\right) \in \mathcal{D}_{<T}$ be such that

$$
\min _{\mathcal{D}<T}(\text { strict })\left(v_{*}-\varphi\right)=\left(v_{*}-\varphi\right)\left(t_{o}, x_{o}\right)=0 ;
$$

we have to show that $L\left(\cdot, \varphi, \partial_{t} \varphi, D \varphi, D^{2} \varphi\right)\left(t_{o}, x_{o}\right) \geq 0$. Suppose to the contrary that we can find $a_{o} \in A$ such that

$$
L^{u_{o}, a_{o}}\left(\cdot, \varphi, \partial_{t} \varphi, D \varphi, D^{2} \varphi\right)\left(t_{o}, x_{o}\right)<0
$$

where $u_{o}:=\hat{u}\left(\cdot, \varphi, \sigma_{X}\left(\cdot, a_{o}\right) D \varphi, a_{o}\right)\left(t_{o}, x_{o}\right)$ and

$$
L^{u, a}(t, x, y, q, p, M):=\mu_{Y}(t, x, y, u, a)-q-\mu_{X}(t, x, a)^{\top} p-\frac{1}{2}\left[\sigma_{X} \sigma_{X}^{\top}(t, x, a) M\right] .
$$

The continuity of $\hat{u}$, cf. Assumption (A) implies that for all $\varepsilon>0$, we can find $\delta>0$ such that $\left|u-u_{o}\right| \leq \varepsilon$ whenever $u \in U$ is such that

$$
\left|\sigma_{Y}\left(t, x, y, u, a_{o}\right)-\sigma_{X}\left(t, x, a_{o}\right) D \varphi(t, x)\right| \leq \delta
$$

for some $(t, x, y) \in \mathcal{D} \times \mathbb{R}$ satisfying $\left|(t, x, y)-\left(t_{o}, x_{o}, \varphi\left(t_{o}, x_{o}\right)\right)\right| \leq \delta$. Recalling the regularity assumptions (2.2) imposed on the coefficients of our controlled dynamics, this implies that we can find $\delta>0$ and an open neighborhood $B \subset \mathcal{D}_{<T}$ of $\left(t_{o}, x_{o}\right)$ such that

$$
\begin{gathered}
L^{u, a_{o}}\left(\cdot, y, \partial_{t} \varphi, D \varphi, D^{2} \varphi\right)(t, x) \leq 0 \quad \forall(t, x) \in B \text { and }(y, u) \in \mathbb{R} \times U \text { s.t. } \\
|y-\varphi(t, x)| \leq \delta \text { and }\left|\sigma_{Y}\left(t, x, y, u, a_{o}\right)-\sigma_{X}\left(t, x, a_{o}\right) D \varphi(t, x)\right| \leq \delta .
\end{gathered}
$$

We now fix $0<\varepsilon<\delta$. It follows from (2.11) that we can find $(t, x, y) \in B \times \mathbb{R}$ satisfying

$$
\varphi(t, x)+\varepsilon>y>v(t, x) .
$$

Then, Theorem 2.3 implies that there exists $\mathfrak{u} \in \mathfrak{U}^{t}$ such that

$$
Y(s \wedge \theta) \geq v_{*}(s \wedge \theta, X(s \wedge \theta)) \text { a.s. } \forall s \geq t,
$$

where we abbreviate $(X, Y):=\left(X_{t, x}^{a_{o}}, Y_{t, x, y}^{\mathfrak{u}, a_{o}}\right)$ and $\theta$ is the stopping time

$$
\theta:=\theta^{1} \wedge \theta^{2}
$$

$\theta^{1}$ is the first exit time of $(\cdot, X)$ from $B$ and $\theta^{2}$ is the first time $|Y-\varphi(\cdot, X)|$ reaches the level $\delta$. Using (2.11) again, we observe that $v_{*}-\varphi \geq 0$ on $B$ and $\left(v_{*}-\varphi\right) \geq \zeta$ on $\partial B$, for some $\zeta>0$. Then, (2.14) and the definition of $\theta$ imply that

$$
\Delta(s):=Y(s \wedge \theta)-\varphi(s \wedge \theta, X(s \wedge \theta)) \geq(\zeta \wedge \delta) \mathbf{1}_{\llbracket \theta, T \rrbracket}(s) \text { a.s. } \forall s \geq t .
$$


We now use (2.12) to obtain a contradiction to (2.15). To this end, set

$$
\begin{aligned}
\lambda & :=\sigma_{Y}\left(\cdot, X, Y, \mathfrak{u}\left[a_{o}\right], a_{o}\right)-\sigma_{X}\left(\cdot, X, a_{o}\right) D \varphi(\cdot, X), \\
\beta & :=\left(L^{\mathfrak{u}\left[a_{o}\right] ., a_{o}}\left(\cdot, Y, \partial_{t} \varphi, D \varphi, D^{2} \varphi\right)(\cdot, X)\right)|\lambda|^{-2} \lambda \mathbf{1}_{\{|\lambda| \geq \delta\}} .
\end{aligned}
$$

It follows from the definition of $\theta$ and our regularity and relative growth conditions (2.2) and (2.3) 2 that $\beta$ is uniformly bounded on $\llbracket t, \theta \rrbracket$. This ensures that the (positive) exponential local martingale $M$ defined by the stochastic differential equation

$$
M(\cdot)=1-\int_{t}^{\cdot \wedge \theta} M(s) \beta_{s}^{\top} d W_{s}
$$

is a true martingale. Recalling (2.12), an application of Itô's formula shows that $M \Delta$ is a local super-martingale. By the definition of $\theta \leq \theta^{2}$, this process is bounded by the martingale $M \delta$, and is therefore a super-martingale. In particular, (2.15) implies that

$$
y-\varphi(t, x)=\Delta(t) \geq \mathbb{E}[M(\theta) \Delta(\theta)] \geq \mathbb{E}[M(\theta)(\zeta \wedge \delta)]=\zeta \wedge \delta .
$$

The required contradiction is obtained by choosing $\varepsilon:=(\zeta \wedge \delta) / 2$ in (2.13).

\subsection{Second part of the dynamic programming principle and subsolution property}

We now turn to the second part of the geometric dynamic programming, GDP2, and thus to the main contribution of this paper. As already mentioned in the Introduction, we cannot rely on an abstract measurable selection argument as in 25. Instead, we construct an almost optimal Markovian strategy that will play the role of a measurable selector in the proof of Theorem 2.7 below. This strategy is defined in (2.16) in terms of a smooth supersolution $w$ of (2.10) having specific properties. The existence of $w$ will be proved in Section 3 by considering a family of controlled forward-backward stochastic differential equations and using the regularization technique of [15]. The arguments in Section 3 require existence and stability properties for the forward-backward stochastic differential equations, which hold under the following condition.

Assumption (C). The map $(t, x, y, z) \in \mathcal{D} \times \mathbb{R} \times \mathbb{R}^{d} \mapsto \mu_{Y}^{\hat{u}}(t, x, y, z, a)$ is continuous and uniformly Lipschitz in $(x, y, z)$, uniformly in $a \in A$, and $(y, z) \in \mathbb{R} \times \mathbb{R}^{d} \mapsto$ $\mu_{Y}^{\hat{u}}(t, x, y, z, a)$ has linear growth, uniformly in $(t, x, a) \in \mathcal{D} \times A$.

We also assume that the comparison principle holds for (2.10); see e.g. [9] for sufficient conditions.

Assumption (D). Let $\underline{w}$ (resp. $\bar{w}$ ) be a lower-semicontinuous (resp. upper-semicontinuous) bounded viscosity supersolution (resp. subsolution) of (2.10). Then, $\underline{w} \geq \bar{w}$ on $\mathcal{D}$.

As in [15], the regularization procedure also requires a concavity property for the operator $L^{a}$.

\footnotetext{
${ }^{2}$ This last condition is missing in $[5]$.
} 
Assumption (E). For all $(t, x, a) \in \mathcal{D} \times A,(y, p) \in \mathbb{R} \times \mathbb{R}^{d} \mapsto L^{a}(t, x, y, 0, p, 0)$ is concave.

We denote by $C_{b}^{\infty}(\mathcal{D})$ the set of bounded functions on $\mathcal{D}$ which have bounded derivatives of all orders. The following lemma summarizes the result of Section 3 in the present context.

Lemma 2.6. Let Assumptions $(\mathbf{A})$ to $(\mathbf{E})$ hold. Let $B \subset \mathcal{D}$ be a compact set and let $\phi$ be a continuous function such that $\phi \geq v_{*}+\eta$ on $B$, for some $\eta>0$. Then, there exists $w \in C_{b}^{\infty}(\mathcal{D})$ such that

(i) $w$ is a classical supersolution of (2.10),

(ii) $w \leq \phi$ on $B$.

Proof. By Theorem 2.5 and Assumption (D) $v_{*}$ dominates any bounded subsolution of (2.10). Thus, the lemma is a special case of Theorem 3.3 below, applied with $\underline{w}:=v_{*}$.

We can now state the main result of this section, the second part of the geometric dynamic programming principle.

Theorem 2.7 (GDP2). Let Assumptions (A) to (E) hold. Fix $(t, x, y)$ in $\mathcal{D} \times \mathbb{R}$, let $B \subset \mathcal{D}$ be a compact set containing $(t, x)$ and let $\theta^{\alpha}$ be the first exit time of $\left(\cdot, X_{t, x}^{\alpha}\right)$ from $B$, for $\alpha \in \mathcal{A}^{t}$. Let $\phi$ be a continuous function such that $\phi \geq v_{*}+\eta$ on $\partial B \backslash \mathcal{D}_{T}$ for some $\eta>0$ and suppose that there exists $\mathfrak{u}_{o} \in \mathfrak{U}^{t}$ such that

$$
Y_{t, x, y}^{\mathfrak{u}_{o}, \alpha}\left(\theta^{\alpha}\right) \geq \phi\left(\theta^{\alpha}, X_{t, x}^{\alpha}\left(\theta^{\alpha}\right)\right) \mathbf{1}_{\left\{\theta^{\alpha}<T\right\}}+g\left(X_{t, x}^{\alpha}(T)\right) \mathbf{1}_{\left\{\theta^{\alpha}=T\right\}} \text { a.s. } \forall \alpha \in \mathcal{A}^{t} .
$$

Then, there exists $\mathfrak{u} \in \mathfrak{U}^{t}$ such that

$$
Y_{t, x, y}^{\mathfrak{u}, \alpha}(T) \geq g\left(X_{t, x}^{\alpha}(T)\right) \text { a.s. } \forall \alpha \in \mathcal{A}^{t},
$$

and in particular $y \geq v(t, x)$.

Proof. Note that the lower-semicontinuity of $v_{*}$ ensures that $\phi \geq v_{*}+\eta$ on the closure of the bounded set $\partial B \backslash \mathcal{D}_{T}$. It then follows from Lemma 2.6 (applied to the closure of $\left.\partial B \backslash \mathcal{D}_{T}\right)$ that we can find a function $w \in C_{b}^{\infty}$ which is a supersolution of (2.10) and satisfies $w \leq \phi$ on $\partial B \backslash \mathcal{D}_{T}$. Next, we introduce $\mathfrak{u} \in \mathfrak{U}^{t}$ satisfying

$$
\mathfrak{u}[\alpha]=\mathfrak{u}_{o}[\alpha] \mathbf{1}_{\llbracket t, \theta^{\alpha} \llbracket}+\mathbf{1}_{\llbracket \theta^{\alpha}, T \rrbracket} \hat{u}\left(\cdot, X_{t, x}^{\alpha}, Y_{t, x, y}^{\mathfrak{u}, \alpha},\left(\sigma_{X}(\cdot, \alpha) D w\right)\left(\cdot, X_{t, x}^{\alpha}\right), \alpha\right)
$$

for $\alpha \in \mathcal{A}^{t}$. To this end, let $Y$ be the unique strong solution of the equation

$$
\begin{aligned}
Y= & Y_{t, x, y}^{\mathfrak{u}_{o}, \alpha}\left(\theta^{\alpha}\right)+\int_{\theta^{\alpha}}^{\theta^{\alpha} \vee \cdot} \mu_{Y}^{\hat{u}}\left(\cdot, Y(s), \sigma_{X}\left(\cdot, \alpha_{s}\right) D w, \alpha_{s}\right)\left(s, X_{t, x}^{\alpha}(s)\right) d s \\
& +\int_{\theta^{\alpha}}^{\theta^{\alpha} \vee \cdot} \sigma_{X}\left(s, X_{t, x}^{\alpha}(s), \alpha_{s}\right) D w\left(s, X_{t, x}^{\alpha}(s)\right) d W_{s}
\end{aligned}
$$

which is well-posed by Assumption (C) Then, if we define $\mathfrak{u}$ by

$$
\mathfrak{u}[\alpha]:=\mathfrak{u}_{o}[\alpha] \mathbf{1}_{\llbracket t, \theta^{\alpha} \llbracket}+\mathbf{1}_{\llbracket \theta^{\alpha}, T \rrbracket} \hat{u}\left(\cdot, X_{t, x}^{\alpha}, Y,\left(\sigma_{X}(\cdot, \alpha) D w\right)\left(\cdot, X_{t, x}^{\alpha}\right), \alpha\right),
$$


it follows via the definition of $\hat{u}$ in Assumption $(\mathbf{A})$ that

$$
\sigma_{X}\left(s, X_{t, x}^{\alpha}(s), \alpha_{s}\right) D w\left(s, X_{t, x}^{\alpha}(s)\right)=\sigma_{Y}\left(s, X_{t, x}^{\alpha}(s), Y(s), \mathfrak{u}[\alpha]_{s}, \alpha_{s}\right)
$$

for $s \geq \theta^{\alpha}$; that is, (2.16) is satisfied. Note that $\mathfrak{u}$ indeed belongs to the set $\mathfrak{U}^{t}$ of nonanticipating strategies, due to the fact that $\theta^{\alpha}=\theta^{\alpha^{\prime}}$ on $\left\{\left.\alpha\right|_{[t, s]}=\left.\alpha^{\prime}\right|_{[t, s]}\right\} \cap\left\{\theta^{\alpha} \leq s\right\}$ for all $s \in[t, T]$ and $\alpha, \alpha^{\prime} \in \mathcal{A}^{t}$.

Let us now fix $\alpha \in \mathcal{A}^{t}$. Since $\phi \geq w$ on $\partial B \backslash \mathcal{D}_{T}$, the definition of the first exit time $\theta^{\alpha}$ implies that

$$
Y_{t, x, y}^{\mathfrak{u}, \alpha}\left(\theta^{\alpha}\right) \geq w\left(\theta^{\alpha}, X_{t, x}^{\alpha}\left(\theta^{\alpha}\right)\right) \mathbf{1}_{\left\{\theta^{\alpha}<T\right\}}+g\left(X_{t, x}^{\alpha}(T)\right) \mathbf{1}_{\left\{\theta^{\alpha}=T\right\}} \text { a.s. }
$$

Applying Itô's formula to the smooth function $w$ and using that it is a supersolution of (2.10) then leads to

$$
Y_{t, x, y}^{\mathfrak{u}, \alpha}(T) \geq w\left(T, X_{t, x}^{\alpha}(T)\right) \mathbf{1}_{\left\{\theta^{\alpha}<T\right\}}+g\left(X_{t, x}^{\alpha}(T)\right) \mathbf{1}_{\left\{\theta^{\alpha}=T\right\}} \geq g\left(X_{t, x}^{\alpha}(T)\right) \text { a.s. }
$$

as claimed. Since $\alpha \in \mathcal{A}^{t}$ was arbitrary, this implies that $y \geq v(t, x)$.

As a consequence of Theorem 2.7. we can now prove that the upper-semicontinuous envelope $v^{*}$ of $v$ is a viscosity subsolution of (2.10).

Theorem 2.8. Let Assumptions (A) to (E) hold. Then, the function $v^{*}$ is a bounded viscosity subsolution of (2.10).

Proof. We remark that due to the present (game) context, the proof in [5] cannot be reproduced per se; see Remark 2.9 for details. Instead, we first consider a case where $\mu_{Y}^{\hat{u}}$ is non-decreasing in $y$ and then treat the general case by reduction.

Step 1. Let $\mu_{Y}^{\hat{u}}$ be non-decreasing in its third variable $y$. We only prove the subsolution property on $\mathcal{D}_{T}$; the subsolution property on $\mathcal{D}_{<T}$ follows from similar arguments based on Theorem 2.7. see [6, Section 3] and [5, Section 5]. Let $x_{o} \in \mathbb{R}^{d}$ be such that

$$
\max _{\mathcal{D}}(\operatorname{strict})\left(v^{*}-\varphi\right)=\left(v^{*}-\varphi\right)\left(T, x_{o}\right)=0
$$

for some smooth function $\varphi$, and suppose for contradiction that

$$
\varphi\left(T, x_{o}\right)-g\left(x_{o}\right)=: 2 \kappa>0 .
$$

Define $\tilde{\varphi}(t, x):=\varphi(t, x)+\sqrt{T-t}$ for $(t, x) \in \mathcal{D}$; then (2.18) implies that there exists $\delta>0$ such that

$$
\tilde{\varphi}-g>\kappa \text { on } B_{\delta}:=\left\{(t, x) \in \mathcal{D}:\left|(t, x)-\left(T, x_{o}\right)\right| \leq \delta\right\} .
$$

Moreover, the fact that $\partial_{t} \tilde{\varphi}(t, x) \rightarrow-\infty$ as $t \rightarrow T$ and the monotonicity assumption on $\mu_{Y}^{\hat{u}}$ imply that, after possibly changing $\delta>0$,

$$
\inf _{a \in A} L^{a}\left(\cdot, y, \partial_{t} \tilde{\varphi}, D \tilde{\varphi}, D^{2} \tilde{\varphi}\right)(t, x) \geq 0, \quad \forall(t, x, y) \in B_{\delta} \times \mathbb{R} \text { s.t. } y \geq \tilde{\varphi}(t, x)-\delta .
$$

Let

$$
-\zeta:=\sup _{\partial B_{\delta} \backslash \mathcal{D}_{T}}\left(v^{*}-\tilde{\varphi}\right)<0
$$


where the strict inequality follows from the fact that $\left(T, x_{o}\right)$ achieves a strict maximum of $v^{*}-\tilde{\varphi}$. Fix $0<\varepsilon<\delta \wedge \zeta \wedge \kappa$ and let $(t, x) \in B_{\delta}$ and $y \in \mathbb{R}$ be such that

$$
-\varepsilon+\tilde{\varphi}(t, x)<y<v(t, x)
$$

see (2.17). Next, consider the strategy defined in a Markovian way by

$$
\alpha \in \mathcal{A}^{t} \mapsto \hat{\mathfrak{u}}[\alpha]:=\hat{u}\left(\cdot, X_{t, x}^{\alpha}, Y_{t, x, y}^{\hat{u}, \alpha},\left(\sigma_{X}(\cdot, \alpha) D \tilde{\varphi}\right)\left(\cdot, X_{t, x}^{\alpha}\right), \alpha\right) .
$$

Without loss of generality, we can assume that $D \tilde{\varphi}$ is bounded on $[t, T] \times \mathbb{R}^{d}$ and then the same arguments as in the proof of Theorem 2.7 show that $\hat{\mathfrak{u}}$ is well-defined as an element of $\mathfrak{U}^{t}$.

Given $\alpha \in \mathcal{A}^{t}$, let $\theta^{\alpha}$ be the first exit time of $\left(\cdot, X_{t, x}^{\alpha}\right)$ from $B_{\delta}$. Then, by the definition of $\hat{u}$ and (2.20), the process $Y_{t, x, y}^{\hat{u}, \alpha}-\tilde{\varphi}\left(\cdot, X_{t, x}^{\alpha}\right)$ is non-decreasing until it reaches $-\delta$ or time reaches $\theta^{\alpha}$. But since this process starts above $-\varepsilon>-\delta$, it is in fact non-decreasing until $\theta^{\alpha}$ and hence

$$
Y_{t, x, y}^{\hat{u}, \alpha}\left(\theta^{\alpha}\right) \geq \tilde{\varphi}\left(\theta^{\alpha}, X_{t, x}^{\alpha}\left(\theta^{\alpha}\right)\right)-\varepsilon .
$$

Set $\eta:=\zeta-\varepsilon>0$. Then, $\phi:=\tilde{\varphi}-\varepsilon \geq v_{*}+\eta$ on the closure of $\partial B_{\delta} \backslash \mathcal{D}_{T}$; see (2.21). Moreover, it follows from (2.19) that $\tilde{\varphi}-\varepsilon \geq g+\kappa-\varepsilon \geq g$ on $B_{\delta} \cap \mathcal{D}_{T}$; in particular,

$$
Y_{t, x, y}^{\hat{u}, \alpha}\left(\theta^{\alpha}\right) \geq \phi\left(\theta^{\alpha}, X_{t, x}^{\alpha}\left(\theta^{\alpha}\right)\right) \mathbf{1}_{\left\{\theta^{\alpha}<T\right\}}+g\left(X_{t, x}^{\alpha}(T)\right) \mathbf{1}_{\left\{\theta^{\alpha}=T\right\}} .
$$

Since $\alpha \in \mathcal{A}^{t}$ was arbitrary, Theorem 2.7 yields a contradiction to the fact that $y<v(t, x)$.

Step 2. We now turn to the general case. Fix $\rho>0$ and define $\tilde{Y}_{t, x, y}^{\mathfrak{u}, \alpha}$ as the strong solution of

$$
d \tilde{Y}(s)=\tilde{\mu}_{Y}\left(s, X_{t, x}^{\alpha}(s), \tilde{Y}(s), \mathfrak{u}[\alpha]_{s}, \alpha_{s}\right) d s+\tilde{\sigma}_{Y}\left(s, X_{t, x}^{\alpha}(s), \tilde{Y}(s), \mathfrak{u}[\alpha]_{s}, \alpha_{s}\right) d W_{s}
$$

with initial data $Y(t)=y$, where

$$
\begin{aligned}
& \tilde{\mu}_{Y}(t, x, y, u, a):=\rho y+e^{\rho t} \mu_{Y}\left(t, x, e^{-\rho t} y, u, a\right), \\
& \tilde{\sigma}_{Y}(t, x, y, u, a):=e^{\rho t} \sigma_{Y}\left(t, x, e^{-\rho t} y, u, a\right) .
\end{aligned}
$$

Set $\tilde{g}:=e^{\rho T} g$ and define

$$
\tilde{v}(t, x):=\inf \left\{y \in \mathbb{R}: \exists \mathfrak{u} \in \mathfrak{U}^{t} \text { s.t. } \tilde{Y}_{t, x, y}^{\mathfrak{u}, \alpha}(T) \geq \tilde{g}\left(X_{t, x}^{\alpha}(T)\right) \text { a.s. } \forall \alpha \in \mathcal{A}^{t}\right\} .
$$

Since $\mu_{Y}^{\hat{u}}$ has linear growth in its second argument $y$, see Assumption $(\mathbf{C})$, one can choose $\rho>0$ so that

$$
\tilde{\mu}_{Y}^{\hat{u}}:(t, x, y, z, a) \mapsto \rho y+e^{\rho t} \mu_{Y}^{\hat{u}}\left(x, e^{-\rho t} y, e^{-\rho t} z, a\right)
$$

is non-decreasing in its $y$-variable. This means that these dynamics satisfy the monotonicity assumption used in Step 1 above; moreover, Assumptions (A) to (E) are also satisfied. Hence, the upper-semicontinuous envelope $\tilde{v}^{*}$ of $\tilde{v}$ is a viscosity subsolution of

$$
\begin{aligned}
& \tilde{L}\left(\cdot, \varphi, \partial_{t} \varphi, D \varphi, D^{2} \varphi\right)=0 \quad \text { on } \quad \mathcal{D}_{<T} \\
& \varphi-\tilde{g}=0 \quad \text { on } \quad \mathcal{D}_{T},
\end{aligned}
$$


where $\tilde{L}$ is defined like $L$ but with $\tilde{\mu}_{Y}$ and $\tilde{\sigma}_{Y}$ instead of $\mu_{Y}$ and $\sigma_{Y}$; that is,

$$
\tilde{L}:=\inf _{a \in A} \tilde{L}^{a}
$$

and

$$
\begin{aligned}
\tilde{L}^{a}(t, x, y, q, p, M):= & \rho y+e^{\rho t} \mu_{Y}^{\tilde{u}}\left(t, x, e^{-\rho t} y, e^{-\rho t} \sigma_{X}(t, x, a) p, a\right) \\
& -q-\mu_{X}(t, x, a)^{\top} p-\frac{1}{2}\left[\sigma_{X} \sigma_{X}^{\top}(t, x, a) M\right],
\end{aligned}
$$

where $\tilde{u}$ is defined like $\hat{u}$ but now in terms of $\tilde{\sigma}_{Y}$. Since $\tilde{v}(t, x)=e^{\rho t} v(t, x)$, this is equivalent to saying that $v^{*}$ is a viscosity subsolution of (2.10).

Remark 2.9. In the proofs of [5, Section 5], the condition $|y-\tilde{\varphi}(t, x)| \leq \delta$ was used instead of $y \geq \tilde{\varphi}(t, x)-\delta$ as in (2.20). Correspondingly, $\theta^{\alpha}$ would then be the minimum of the first time when $\left(\cdot, X_{t, x}^{\alpha}\right)$ reaches the boundary of $B_{\delta}$ and the first time when $\left|Y_{t, x, y}^{\hat{u}, \alpha}-\tilde{\varphi}\left(\cdot, X_{t, x}^{\alpha}\right)\right|$ reaches $\delta$. In this case, similar arguments as above imply that

$$
Y_{t, x, y}^{\hat{\mathfrak{u}}, \alpha}\left(\theta^{\alpha}\right) \geq \phi\left(\theta^{\alpha}, X_{t, x}^{\alpha}\left(\theta^{\alpha}\right)\right) \mathbf{1}_{\left\{\theta^{\alpha}<T\right\}}+g\left(X_{t, x}^{\alpha}(T)\right) \mathbf{1}_{\left\{\theta^{\alpha}=T\right\}}
$$

and in the context of [5], this was enough to obtain a contradiction. However, it is not the case in our situation: this stopping time $\theta^{\alpha}$ is not an exit time of $\left(\cdot, X_{t, x}^{\alpha}\right)$, which is a key condition in our Theorem 2.7

Remark 2.10. It follows from Assumption (D) Theorem 2.5 and Theorem 2.8 that $v$ is continuous and that $v$ is the unique bounded (discontinuous) viscosity solution of (2.10), whenever Assumptions (A) to (E) hold.

\section{On the regularization of concave non-linear PDEs}

In this section, we prove Lemma 2.6 above. We consider a more general setting in order to isolate the result from the particular context of the preceding section; the general version of Lemma 2.6 is stated in Theorem 3.3 below. Our result is very much in the spirit of [15, Theorem 2.1] (see also [2]), which we extend to our setting. Consider the parabolic equation

$$
H\left(\cdot, \varphi, \partial_{t} \varphi, D \varphi, D^{2} \varphi\right)=0 \text { on } \mathcal{D}_{<T} \text { and } \varphi=g \text { on } \mathcal{D}_{T},
$$

where, for $(y, q, p, M) \in \mathbb{R} \times \mathbb{R} \times \mathbb{R}^{d} \times \mathbb{M}^{d}$,

$H(\cdot, y, q, p, M):=\inf _{a \in A}\left(f\left(\cdot, y, \sigma_{X}(\cdot, a) p, a\right)-q-\mu_{X}(\cdot, a)^{\top} p-\frac{1}{2} \operatorname{Tr}\left[\sigma_{X} \sigma_{X}^{\top}(\cdot, a) M\right]\right)$,

for some continuous function $f: \mathcal{D} \times \mathbb{R} \times \mathbb{R}^{d} \times A \rightarrow \mathbb{R}$ such that

$$
\begin{gathered}
(y, z) \mapsto f(t, x, y, z, a) \text { is concave and has linear growth } \\
\text { uniformly in }(t, x, a) \in \mathcal{D} \times A, \\
(t, x, y, z) \mapsto f(t, x, y, z, a) \text { is continuous and uniformly Lipschitz in }(x, y, z), \\
\text { uniformly in } a \in A .
\end{gathered}
$$

We continue to assume that the continuity and growth conditions (2.2) hold for $\mu_{X}$ and $\sigma_{X}$, and that $g$ is bounded and Lipschitz continuous. 
Our aim is to provide a smooth supersolution of (3.1) which is controlled by a given viscosity supersolution of the same equation, in the sense of Theorem 3.3 below. To this end, we first introduce a family of supersolutions of perturbations of (3.1). Namely, they correspond to the shaken coefficients in the terminology of [15]; i.e. to the operators $H_{\varepsilon}$ defined for $(t, x, y, q, p, M) \in \mathcal{D} \times \mathbb{R} \times \mathbb{R} \times \mathbb{R}^{d} \times \mathbb{M}^{d}$ by

$$
H_{\varepsilon}(t, x, y, q, p, M):=\inf _{b \in B_{\varepsilon}(0)} H((t, x)+b, y, q, p, M), \quad \varepsilon>0,
$$

where $B_{\varepsilon}(0) \subset \mathbb{R}^{d+1}$ denotes the closed ball of radius $\varepsilon$ around the origin. This is also the first step in the analysis of [15, Theorem 2.1], where $f$ does not depend on $y$ and $z$ and the result is obtained by considering a family of standard optimal control problems in the so-called Bolza form. We shall obtain the extension to our framework by considering instead controlled forward-backward stochastic differential equations.

As in the previous section, we impose that the comparison principle holds for (3.1); this will ensure that the family of supersolutions associated to $H_{\varepsilon}$ is controlled from above by the supersolutions of (3.1):

Assumption (F). Let $\underline{w}$ (resp. $\bar{w}$ ) be a lower-semicontinuous (resp. upper-semicontinuous) bounded viscosity supersolution (resp. subsolution) of (3.1). Then, $\underline{w} \geq \bar{w}$ on $\mathcal{D}$.

Proposition 3.1. Let Assumption $(\mathbf{F})$ hold. For all $\varepsilon \in[0,1]$, there exists a bounded and continuous viscosity solution $w_{\varepsilon}$ of

$$
H_{\varepsilon}\left(\cdot, \varphi, \partial_{t} \varphi, D \varphi, D^{2} \varphi\right)=0 \text { on } \mathcal{D}_{<T}
$$

with the following properties:

(i) There exists $c^{\varepsilon} \in[0, T)$ such that

$$
w_{\varepsilon} \geq g+\varepsilon \text { on }\left[T-c^{\varepsilon}, T\right] \times \mathbb{R}^{d} \text { and } c^{\varepsilon}>0 \text { if } \varepsilon>0 .
$$

(ii) For any compact set $B \subset \mathcal{D}$, there exists $c_{\varepsilon}^{B}$ satisfying $c_{\varepsilon}^{B} \rightarrow 0$ as $\varepsilon \rightarrow 0$ and

$$
w_{\varepsilon}-c_{\varepsilon}^{B} \leq w_{0} \text { on } B .
$$

Proof. We shall construct the functions $w_{\varepsilon}$ as value functions associated to controlled forward-backward stochastic differential equations. Some of the subsequent technical arguments are known and will only be sketched; we focus on the points specific to our problem.

Step 1. In this step, we introduce a family of controlled forward-backward equations indexed by $\varepsilon$. To this end, let $\mathcal{T}$ denote the set of stopping times and $\mathbf{D}_{2}$ the collection of pairs $(\tau, \xi)$ such that $\tau \in \mathcal{T}$ and $\xi \in L^{2}\left(\mathcal{F}_{\tau} ; \mathbb{R}^{d}\right)$. Moreover, we denote by $\mathbf{S}_{2}$ the set of predictable processes $\psi$ such that $\sup _{t \leq T}\left|\psi_{t}\right| \in L^{2}$ and by $\mathbf{H}_{2}$ the set of $\mathbb{P} \otimes d t$-square-integrable predictable processes. Finally, we let $\mathcal{B}_{\varepsilon}$ (resp. $\mathcal{A})$ denote the set of predictable processes with values in the ball $B_{\varepsilon}(0) \subset \mathbb{R}^{d+1}$ (resp. A). For each $\varepsilon \in[0,1]$, given a control $\gamma:=(\alpha, \beta) \in \mathcal{A} \times \mathcal{B}_{\varepsilon}=: \Gamma_{\varepsilon}$ and an initial condition $\zeta:=(\tau, \xi) \in \mathbf{D}_{2}$, we now consider the decoupled forward-backward stochastic differential equation

$$
\begin{aligned}
\bar{X}^{\zeta, \gamma} & =\xi+\int_{\tau}^{.} \mu_{X}\left(\left(s, \bar{X}_{s}^{\zeta, \gamma}\right)+\beta_{s}, \alpha_{s}\right) d s+\int_{\tau}^{\cdot} \sigma_{X}\left(\left(s, \bar{X}_{s}^{\zeta, \gamma}\right)+\beta_{s}, \alpha_{s}\right) d W_{s} \\
\bar{Y}^{\varepsilon, \zeta, \gamma} & =g_{\varepsilon}\left(\bar{X}_{T}^{\zeta, \gamma}\right)-\int_{\cdot \vee \tau}^{T} f_{\gamma}^{\zeta}\left(s, \bar{Y}_{s}^{\varepsilon, \zeta, \gamma}, \bar{Z}_{s}^{\varepsilon, \zeta, \gamma}\right) d s-\int_{\cdot \vee \tau}^{T} \bar{Z}_{s}^{\varepsilon, \zeta, \gamma} d W_{s},
\end{aligned}
$$


where $g_{\varepsilon}:=g+2 \varepsilon$ and

$$
f_{\gamma}^{\zeta}(s, y, z):=f\left(\left(s, \bar{X}_{s}^{\zeta, \gamma}\right)+\beta_{s}, y, z, \alpha_{s}\right) \text { for }(s, y, z) \in[0, T] \times \mathbb{R} \times \mathbb{R}^{d} .
$$

Note that the forward equation does not depend on $\varepsilon$. Moreover, (3.2) implies that

$$
\begin{gathered}
(y, z) \in \mathbb{R} \times \mathbb{R}^{d} \mapsto f_{\gamma}^{\zeta}(s, y, z) \text { is Lipschitz continuous with linear growth } \\
\text { uniformly in }(s, \zeta, \gamma) \in[0, T] \times \mathbf{D}_{2} \times \Gamma_{1} .
\end{gathered}
$$

Existence and uniqueness of the solution $\left(\bar{X}^{\zeta, \gamma}, \bar{Y}^{\varepsilon, \zeta, \gamma}, \bar{Z}^{\varepsilon, \zeta, \gamma}\right) \in \mathbf{S}_{2} \times \mathbf{S}_{2} \times \mathbf{H}_{2}$ are standard; see e.g. [18. For $\gamma \in \Gamma_{\varepsilon}, \zeta=(\tau, \xi) \in \mathbf{D}_{2}$ and $\theta \in \mathcal{T}$ such that $\theta \geq \tau$ a.s., we now define

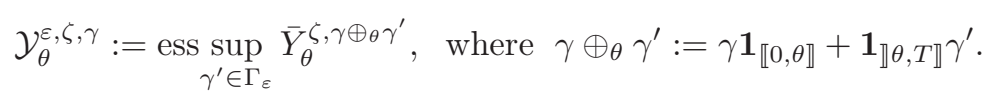

By a Girsanov change of measure argument as in [8, Proposition 3.3] (which is in turn an extension of [21]), it follows that

$$
(t, x) \in \mathcal{D} \mapsto w_{\varepsilon}(t, x):=\mathcal{Y}_{t}^{\varepsilon,(t, x), \gamma}
$$

is a deterministic function on $\mathcal{D}$, independent of $\gamma \in \Gamma_{1}$. In the remainder of the proof, we show that $w_{\varepsilon}$ satisfies the requirements of the proposition.

Step 2. We provide some estimates that will be used later on. We first observe that (3.2), 18, Theorem 1.5], (2.2) and the Lipschitz continuity of $g$ imply that we can find $c>0$, independent of $\varepsilon$, such that

$$
\mathbb{E}\left[\left|\bar{Y}_{\tau_{1}}^{\ell, \zeta_{1}, \gamma \oplus_{\tau_{1}} \gamma^{\prime}}-\mathbb{E}_{\tau_{1}}\left[\bar{Y}_{\tau_{2}}^{\ell, \zeta_{2}, \gamma \oplus_{\tau_{2}} \gamma^{\prime}}\right]\right|^{2}\right] \leq c \mathbb{E}\left[\left|\xi_{1}-\xi_{2}\right|^{2}+\left|\tau_{1}-\tau_{2}\right|\right]
$$

for all $\zeta_{i}=\left(\tau_{i}, \xi_{i}\right) \in \mathbf{D}_{2}, i=1,2$, such that $\tau_{1} \leq \tau_{2}$, and $\gamma, \gamma^{\prime} \in \Gamma_{\varepsilon}$. As the family $\left\{\bar{Y}_{\tau_{1}}^{\varepsilon, \zeta_{1}, \gamma \oplus_{\tau_{1}} \gamma^{\prime}}\right\}_{\gamma^{\prime} \in \Gamma_{\varepsilon}}$ is directed upward, it follows from [17, Proposition VI-1-1] that we can find a sequence $\left(\gamma_{n}^{\prime}\right)_{n} \subset \Gamma_{\varepsilon}$ such that $\bar{Y}_{\tau_{1}}^{\varepsilon, \zeta_{1}, \gamma \oplus \tau_{\tau_{1}} \gamma_{n}^{\prime}} \uparrow \mathcal{Y}_{\tau_{1}}^{\varepsilon, \zeta_{1}, \gamma}$ a.s.; see e.g. [6], Lemma 2.4] for a similar argument. Then

$$
\mathcal{Y}_{\tau_{1}}^{\varepsilon, \zeta_{1}, \gamma}-\mathbb{E}_{\tau_{1}}\left[\mathcal{Y}_{\tau_{2}}^{\varepsilon, \zeta_{2}, \gamma}\right] \leq \liminf _{n}\left(\bar{Y}_{\tau_{1}}^{\varepsilon, \zeta_{1}, \gamma \oplus_{\tau_{1}} \gamma_{n}^{\prime}}-\mathbb{E}_{\tau_{1}}\left[\bar{Y}_{\tau_{2}}^{\varepsilon, \zeta_{2}, \gamma \oplus_{\tau_{2}} \gamma_{n}^{\prime}}\right]\right) .
$$

Similarly, we can find $\left(\gamma_{n}^{\prime \prime}\right)_{n} \subset \Gamma_{\varepsilon}$ such that

$$
\mathbb{E}_{\tau_{1}}\left[\mathcal{Y}_{\tau_{2}}^{\varepsilon, \zeta_{2}, \gamma}\right]-\mathcal{Y}_{\tau_{1}}^{\varepsilon, \zeta_{1}, \gamma} \leq \liminf _{n}\left(\mathbb{E}_{\tau_{1}}\left[\bar{Y}_{\tau_{2}}^{\varepsilon, \zeta_{2}, \gamma \oplus_{\tau_{2}} \gamma_{n}^{\prime \prime}}\right]-\bar{Y}_{\tau_{1}}^{\varepsilon, \zeta_{1}, \gamma \oplus_{\tau_{1}} \gamma_{n}^{\prime \prime}}\right) .
$$

Moreover, as $g$ is bounded and $f$ satisfies (3.6), we deduce from [20, Theorem 2.2] that

$$
\underset{\gamma^{\prime} \in \Gamma_{\varepsilon}}{\operatorname{ess} \sup _{\tau_{2}}}\left(\left|\bar{Y}_{\tau_{2}}^{\zeta_{2}, \gamma^{\prime}}\right|+\left|\bar{Y}_{\tau_{1}}^{\zeta_{1}, \gamma^{\prime}}\right|\right) \in L^{2} .
$$

Then, combining the above with the dominated convergence theorem and the apriori estimate (3.9) yields that

$$
\mathbb{E}\left[\left|\mathcal{Y}_{\tau_{1}}^{\varepsilon, \zeta_{1}, \gamma}-\mathbb{E}_{\tau_{1}}\left[\mathcal{Y}_{\tau_{2}}^{\varepsilon, \zeta_{2}, \gamma}\right]\right|^{2}\right] \leq c \mathbb{E}\left[\left|\xi_{1}-\xi_{2}\right|^{2}+\left|\tau_{1}-\tau_{2}\right|\right] .
$$

Applying this inequality to $\zeta_{1}=(t, x) \in \mathcal{D}$ and $\zeta_{2}=(T, x)$, we see that

$$
\left|\mathcal{Y}_{t}^{\varepsilon,(t, x), \gamma}-g_{\varepsilon}(x)\right| \leq c \sqrt{T-t} \quad \forall(t, x, \gamma) \in \mathcal{D} \times \Gamma_{\varepsilon} .
$$


Step 3. The fact that $w_{\varepsilon}(z)=\mathcal{Y}_{t}^{\varepsilon, z, \gamma}$ for all $z=(t, x) \in \mathcal{D}$ ensures that $w_{\varepsilon}(\zeta)=\mathcal{Y}_{\tau}^{\varepsilon, \zeta, \gamma}$ for simple random variables $\zeta=(\tau, \xi) \in \mathbf{D}_{2}$. The estimate (3.10) shows that the function $w_{\varepsilon}$ is continuous and a simple approximation argument implies that $w_{\varepsilon}(\zeta)=\mathcal{Y}_{\tau}^{\varepsilon, \zeta, \gamma}$ for all $\zeta=(\tau, \xi) \in \mathbf{D}_{2}$ and $\gamma \in \Gamma_{\varepsilon}$. In particular, for $z=(t, x) \in \mathcal{D}$,

$$
w_{\varepsilon}\left(\tau, \bar{X}_{\tau}^{z, \gamma}\right)=\mathcal{Y}_{\tau}^{\varepsilon, z, \gamma} \text { for all } \tau \in \mathcal{T}_{t},
$$

and $\mathcal{Y}^{\varepsilon, z, \gamma}$ admits a modification with continuous paths.

Step 4. We now sketch the proof of the dynamic programming principle for $\mathcal{Y}^{\varepsilon}$, and thus for $w_{\varepsilon}$. Fix $z=(t, x) \in \mathcal{D}, \gamma \in \Gamma_{\varepsilon}$ and let $t \leq \tau \leq \theta \leq T$ be two stopping times. Let $\gamma_{n} \in \Gamma_{\varepsilon}$ be such that $\mathcal{Y}_{\theta}^{\varepsilon, z, \gamma} \leq \bar{Y}_{\theta}^{z, \gamma \oplus_{\theta} \gamma_{n}}+n^{-1}$. Then, by the stability result [18, Theorem 1.5], one can find $c>0$ and a sequence $\left(\delta_{n}\right)_{n \geq 1}$ of random variables converging to 0 a.s. such that

$$
\mathcal{Y}_{\tau}^{\varepsilon, z, \gamma} \geq \bar{Y}_{\tau}^{\varepsilon, z, \gamma \oplus_{\theta} \gamma_{n}}=\mathcal{E}_{\tau, \theta}^{f_{\gamma}^{z}}\left[\bar{Y}_{\theta}^{\varepsilon, z, \gamma \oplus_{\theta} \gamma_{n}}\right] \geq \mathcal{E}_{\tau, \theta}^{f_{\gamma}^{z}}\left[\mathcal{Y}_{\theta}^{\varepsilon, z, \gamma}\right]-c \delta_{n},
$$

where $\mathcal{E}_{\tau, \theta}^{f_{\gamma}^{z}}[\chi]$ denotes the value at time $\tau$ of the first component of the solution to the backward stochastic differential equation with driver $f_{\gamma}^{z}$ and terminal condition $\chi \in L^{2}\left(\mathcal{F}_{\theta}\right)$ at time $\theta$. By sending $n \rightarrow \infty$, this implies that

$$
\mathcal{Y}_{\tau}^{\varepsilon, z, \gamma} \geq \mathcal{E}_{\tau, \theta}^{f_{\gamma}^{z}}\left[\mathcal{Y}_{\theta}^{\varepsilon, z, \gamma}\right]
$$

Conversely, it follows from the comparison principle [18, Theorem 1.6] that

$$
\mathcal{Y}_{\tau}^{\varepsilon, z, \gamma}=\operatorname{ess} \sup _{\gamma^{\prime} \in \Gamma_{\varepsilon}} \bar{Y}_{\tau}^{\varepsilon, z, \gamma \oplus_{\tau} \gamma^{\prime}} \leq \operatorname{ess} \sup _{\gamma^{\prime} \in \Gamma_{\varepsilon}} \mathcal{E}_{\tau, \theta}^{f_{\gamma \oplus \tau}^{z}}\left[\mathcal{Y}_{\theta}^{\varepsilon, z, \gamma \oplus_{\tau} \gamma^{\prime}}\right]
$$

which, combined with the above, implies

$$
\mathcal{Y}_{\tau}^{\varepsilon, z, \gamma}=\operatorname{ess} \sup _{\gamma^{\prime} \in \Gamma_{\varepsilon}} \mathcal{E}_{\tau, \theta}^{f_{\gamma \oplus_{\tau} \gamma^{\prime}}^{z}}\left[\mathcal{Y}_{\theta}^{\varepsilon, z, \gamma \oplus_{\tau} \gamma^{\prime}}\right]
$$

Step 5. We already know from Step 3 that $w_{\varepsilon}$ is continuous. Then, standard arguments based on the identification (3.12) and the dynamic programming principle (3.13) show that $w_{\varepsilon}$ is a continuous viscosity solution of (3.3); see [19] and [8] for details.

Step 6. We conclude with the remaining estimates. The bound (3.11) and the fact that $g$ is bounded imply that $\left(w_{\varepsilon}\right)_{\varepsilon \in[0,1]}$ is uniformly bounded. Moreover, the estimate (3.11) implies (3.4); it remains to prove (3.5). We first observe that the family $\left(w_{\varepsilon}\right)_{\varepsilon \in[0,1]}$ is non-decreasing by the comparison principle for backward stochastic differential equations and therefore admits a limit $\bar{w}_{0} \geq w_{0}$ as $\varepsilon \rightarrow 0$. The stability of viscosity solutions, see e.g. [3], combined with Assumption (F) ensures that $\bar{w}_{0}$ is a continuous bounded viscosity solution of (3.1). Since $w_{0}$ is a bounded continuous viscosity solution of the same equation, Assumption (F) implies that $\lim _{\varepsilon \downarrow 0} w_{\varepsilon}=\bar{w}_{0}=w_{0}$. By Dini's theorem, the convergence is uniform on compact sets, which is (3.5).

Remark 3.2. Consider again the setting of Section 2 with Assumptions $(\mathbf{A})$ to $(\mathbf{E})$ For $f:=\mu_{Y}^{\hat{u}}$, the equations (3.1) and (2.10) coincide and hence $v=w_{0}$ by uniqueness, where $w_{0}$ is defined in (3.8). Thus, the controlled forward-backward stochastic differential equation (3.7) with $\varepsilon=0$ is a dual problem for the stochastic target game. 
We now apply the smoothing technique used in [15, Section 2] to the functions $w_{\varepsilon}$ obtained in Proposition 3.1 to construct a suitable smooth supersolution of (3.1). The stability results (3.4) and (3.5) play an important role in ensuring that the correct boundary condition at time $T$ is satisfied and that the upper bound in (ii) below holds true.

Theorem 3.3. Let Assumption $(\mathbf{F})$ hold and let $\underline{w}$ be a function on $\mathcal{D}$ which dominates any bounded viscosity subsolution of (3.1). Moreover, let $B \subset \mathcal{D}$ be a compact set and $\phi$ be a continuous function such that $\phi \geq \underline{w}+\eta$ on $B$, for some $\eta>0$. Then, there exists $w \in C_{b}^{\infty}(\mathcal{D})$ such that

(i) $w$ is a classical supersolution of (3.1),

(ii) $w \leq \phi$ on $B$.

Proof. The proof is provided for the sake of completeness; we follow closely 15 and [13]. Throughout, any function $w$ on $\mathcal{D}$ is extended to $\mathbb{R} \times \mathbb{R}^{d}$ by $w(t, x)=$ $w(0, x)$ for $t<0$ and $w(t, x)=w(T, x)$ for $t>T$.

Step 1. We first construct a semi-concave function which is a.e. a supersolution of (3.1) in the interior of the parabolic domain. Let $w_{\varepsilon}$ be as in Proposition 3.1. For $k \geq 1$, consider the quadratic inf-convolution

$$
w_{\varepsilon}^{k}(z):=\inf _{z^{\prime} \in \mathcal{D}}\left(w_{\varepsilon}\left(z^{\prime}\right)+k\left|z-z^{\prime}\right|^{2}\right), z \in \mathcal{D} .
$$

Since $w_{\varepsilon}$ is continuous and bounded, the infimum is achieved at some point $\hat{z}_{k}(z)$. Note that $w_{\varepsilon} \geq w_{\varepsilon}^{k} \geq-\left|w_{\varepsilon}\right|_{\infty}$, where $|\cdot|_{\infty}$ denotes the sup-norm on $\mathcal{D}$. Hence,

$$
k\left|z-\hat{z}_{k}(z)\right|^{2}=w_{\varepsilon}^{k}(z)-w_{\varepsilon}\left(\hat{z}_{k}(z)\right) \leq 2\left|w_{\varepsilon}\right|_{\infty}=: l .
$$

It follows that

$$
\left|z-\hat{z}_{k}(z)\right|^{2} \leq l / k=:\left(\rho_{k}\right)^{2}
$$

In particular, $\hat{z}_{k}(z) \rightarrow z$ as $k \rightarrow \infty$ and thus, using again $w_{\varepsilon} \geq w_{\varepsilon}^{k}$ and the continuity of $w_{\varepsilon}$,

$$
w_{\varepsilon}(z) \leq \liminf _{k \rightarrow \infty}\left(w_{\varepsilon}\left(\hat{z}_{k}(z)\right)+k\left|z-\hat{z}_{k}(z)\right|^{2}\right) \leq \limsup _{k \rightarrow \infty} w_{\varepsilon}^{k}\left(\hat{z}_{k}(z)\right) \leq w_{\varepsilon}(z) .
$$

Let $\varphi$ be a smooth function on $\mathcal{D}$ and let $z \in \mathcal{D}_{<T}$ be such that

$$
\min _{\mathcal{D}}\left(w_{\varepsilon}^{k}-\varphi\right)=\left(w_{\varepsilon}^{k}-\varphi\right)(z)=0 .
$$

Then, for any $z^{\prime} \in \mathcal{D}$,

$$
\begin{aligned}
w_{\varepsilon}\left(\hat{z}_{k}(z)+z^{\prime}-z\right)+k\left|\hat{z}_{k}(z)-z\right|^{2}-\varphi\left(z^{\prime}\right) & \geq w_{\varepsilon}^{k}\left(z^{\prime}\right)-\varphi\left(z^{\prime}\right) \\
& \geq w_{\varepsilon}^{k}(z)-\varphi(z) \\
& =w_{\varepsilon}\left(\hat{z}_{k}(z)\right)+k\left|\hat{z}_{k}(z)-z\right|^{2}-\varphi(z) .
\end{aligned}
$$

Hence, the minimum of $z^{\prime} \in \mathcal{D} \mapsto w_{\varepsilon}\left(\hat{z}_{k}(z)+z^{\prime}-z\right)-\varphi\left(z^{\prime}\right)$ is achieved by $z^{\prime}=z$ and therefore

$$
\left(\partial_{t} \varphi, D \varphi, D^{2} \varphi\right)(z) \in \overline{\mathcal{P}}^{-} w_{\varepsilon}\left(\hat{z}_{k}(z)\right),
$$


where $\overline{\mathcal{P}}^{-} w_{\varepsilon}\left(\hat{z}_{k}(z)\right)$ denotes the closed parabolic subjet of $w_{\varepsilon}$ at $\hat{z}_{k}(z)$; see e.g. 9]. In view of Proposition 3.1 this shows that $w_{\varepsilon}^{k}$ is a viscosity supersolution of

$$
H_{\varepsilon}\left(\hat{z}_{k}(\cdot), \varphi, \partial_{t} \varphi, D \varphi, D^{2} \varphi\right) \geq 0 \text { on }\left[\rho_{k}, T-\rho_{k}\right] \times \mathbb{R}^{d} .
$$

Take $k$ large enough so that $\rho_{k} \leq \varepsilon / 2$. Then, (3.14) yields that

$$
\left\{\hat{z}_{k}(z)+b, b \in B_{\varepsilon}(0)\right\} \supset\left\{z+b, b \in B_{\varepsilon / 2}(0)\right\} .
$$

This implies that $w_{\varepsilon}^{k}$ is a viscosity supersolution of

$$
\min _{b \in B_{\varepsilon / 2}(0)} H\left(\cdot+b, \varphi, \partial_{t} \varphi, D \varphi, D^{2} \varphi\right) \geq 0 \text { on }\left[\rho_{k}, T-\rho_{k}\right] \times \mathbb{R}^{d} .
$$

Step 2. We now argue as in [13] to construct from the previous step a smooth supersolution in the interior of the parabolic domain. Since $w_{\varepsilon}^{k}$ is semi-concave, there exist $D_{2}^{a b s} w_{\varepsilon}^{k} \in L^{1}(\mathcal{D})$ and a Lebesgue-singular negative Radon measure $D_{2}^{\text {sing }} w_{\varepsilon}^{k}$ on $\mathcal{D}$ such that

$$
D^{2} w_{\varepsilon}^{k}(d z)=D_{a b s}^{2} w_{\varepsilon}^{k} d z+D_{\text {sing }}^{2} w_{\varepsilon}^{k} \text { in the distribution sense }
$$

and

$$
\left(\partial_{t} w_{\varepsilon}^{k}, D w_{\varepsilon}^{k}, D_{a b s}^{2} w_{\varepsilon}^{k}\right) \in \overline{\mathcal{P}}^{-} w_{\varepsilon}^{k} \text { a.e. on }\left[\rho_{k}, T-\rho_{k}\right] \times \mathbb{R}^{d} ;
$$

see [14, Section 3]. Hence, Step 1 implies that

$$
\min _{b \in B_{\varepsilon / 2}(0)} H\left(\cdot+b, w_{\varepsilon}^{k}, \partial_{t} w_{\varepsilon}^{k}, D w_{\varepsilon}^{k}, D_{a b s}^{2} w_{\varepsilon}^{k}\right) \geq 0 \text { a.e. on }\left[\rho_{k}, T-\rho_{k}\right] \times \mathbb{R}^{d} .
$$

Next, we mollify $w_{\varepsilon}^{k}$. Let $\psi \in C_{b}^{\infty}$ be a non-negative function with support $[-1,0] \times[-1,1]^{d}$ such that $\int_{\mathbb{R}^{d+1}} \psi(z) d z=1$, and set $\psi_{\delta}(z)=\delta^{-d-1} \phi(z / \delta)$ for $z \in \mathbb{R}^{d+1}$ and $\delta>0$. For any bounded measurable function $w$ on $\mathcal{D}$ and $(t, x) \in$ $[\delta, T] \times \mathbb{R}^{d}$, let

$$
\begin{aligned}
& w \star \psi_{\delta}(t, x):=\int_{{\mathbb{R} \times \mathbb{R}^{d}}^{d}} w\left(t^{\prime}, x^{\prime}\right) \psi_{\delta}\left(t^{\prime}-t, x^{\prime}-x\right) d t^{\prime} d x^{\prime} \\
& =\int_{\mathbb{R} \times \mathbb{R}^{d}} w\left(t+t^{\prime}, x+x^{\prime}\right) \psi_{\delta}\left(t^{\prime}, x^{\prime}\right) d t^{\prime} d x^{\prime} .
\end{aligned}
$$

Using (3.15) and the fact that $(y, q, p, M) \in \mathbb{R} \times \mathbb{R} \times \mathbb{R}^{d} \times \mathbb{M}^{d} \mapsto H(\cdot, y, q, p, M)$ is concave due to (3.2), we obtain for $\delta<\varepsilon / 2$ that

$$
H_{0}\left(\cdot, w_{\varepsilon}^{k} \star \psi_{\delta}, \partial_{t} w_{\varepsilon}^{k} \star \psi_{\delta},\left(D w_{\varepsilon}^{k}\right) \star \psi_{\delta},\left(D_{a b s}^{2} w_{\varepsilon}^{k}\right) \star \psi_{\delta}\right)\left(z_{o}\right) \geq 0
$$

for all $z_{o} \in\left[\rho_{k}+\delta, T-\rho_{k}\right] \times \mathbb{R}^{d}$. Note that, since $D_{\text {sing }}^{2} w_{\varepsilon}^{k} \leq 0$ and $\psi_{\delta} \geq 0$,

$$
\begin{aligned}
\int_{\mathbb{R} \times \mathbb{R}^{d}} \psi_{\delta} D_{a b s}^{2} w_{\varepsilon}^{k} d z & \geq \int_{\mathbb{R} \times \mathbb{R}^{d}} \psi_{\delta} D_{a b s}^{2} w_{\varepsilon}^{k} d z+\int_{\mathbb{R} \times \mathbb{R}^{d}} \psi_{\delta} D_{\text {sing }}^{2} w_{\varepsilon}^{k}(d z) \\
& =\int_{\mathbb{R} \times \mathbb{R}^{d}} w_{\varepsilon}^{k} D^{2} \psi_{\delta} d z
\end{aligned}
$$

by integration-by-parts. Since $L$ is parabolic, this shows that, on $\left[\rho_{k}+\delta, T-\rho_{k}\right] \times \mathbb{R}^{d}$,

$$
H_{0}\left(\cdot, w_{\varepsilon}^{k} \star \psi_{\delta}, \partial_{t}\left(w_{\varepsilon}^{k} \star \psi_{\delta}\right), D\left(w_{\varepsilon}^{k} \star \psi_{\delta}\right), D^{2}\left(w_{\varepsilon}^{k} \star \psi_{\delta}\right)\right) \geq 0 .
$$


By sending $k \rightarrow \infty$, we obtain that $w_{\varepsilon, \delta}:=w_{\varepsilon} \star \psi_{\delta}$ is a supersolution of (3.1) on $(\delta, T)$. Moreover, $w_{\varepsilon, \delta} \in C_{b}^{\infty}(\mathcal{D})$ since $\psi \in C_{b}^{\infty}(\mathcal{D})$ and $w_{\varepsilon}$ is bounded by Proposition 3.1

Step 3. We now choose $\varepsilon$ and $\delta$ so that the supersolution property holds at $T$ and (ii) of the theorem is satisfied for $w=w_{\varepsilon, \delta}$. We first use that $\phi \geq \underline{w}+\eta$ and that $\underline{w}$ dominates bounded subsolutions of (3.1); in particular, $\underline{w} \geq w_{0}$ by Proposition 3.1. Choose $\varepsilon$ such that the constant $c_{\varepsilon}^{B}$ in (3.5) satisfies $c_{\varepsilon}^{\bar{B}} \leq \eta / 2$, then

$$
w_{\varepsilon} \leq w_{0}+c_{\varepsilon}^{B} \leq \phi-\eta+c_{\varepsilon}^{B} \leq \phi-\eta / 2 .
$$

As $w_{\varepsilon}$ is continuous, we have $w_{\varepsilon, \delta} \rightarrow w_{\varepsilon}$ uniformly on the compact set $B$. Thus, for $\delta>0$ small enough with respect to $\eta$,

$$
w_{\varepsilon, \delta} \leq \phi \text { on } B
$$

which is assertion (ii). For the supersolution property at $T$, we first appeal to (3.4) and choose $\delta \in\left(0, c^{\varepsilon}\right)$ such that we also have $w_{\varepsilon, \delta} \geq g \star \psi_{\delta}+\varepsilon$ on $\mathcal{D}_{T}$. Since $g$ is uniformly continuous, we can choose $\delta>0$ small enough so that $g \star \psi_{\delta} \geq g-\varepsilon$ on $\mathcal{D}_{T}$, and therefore $w_{\varepsilon, \delta} \geq g$ on the parabolic time boundary.

Step 4. The fact that $w_{\varepsilon, \delta}$ is only a supersolution of (3.1) on $(\delta, T]$ and not on $[0, T]$ is not restrictive; we can follow the same arguments on $[-1, T]$ instead of $[0, T]$ and obtain that $w_{\varepsilon, \delta}$ is a supersolution of (3.1) on $(-1+\delta, T] \supset[0, T]$ for $0<\delta<1$.

Remark 3.4. In some applications, one might want to restrict the spatial domain to an open set $\mathcal{O} \subset \mathbb{R}^{d}$, for instance if the process $X^{\alpha}$ of Section 2 is defined as a stochastic exponential. Then, the equation (3.1) should be naturally set on $\mathcal{D}:=[0, T] \times \mathcal{O}$. In this more general context, the arguments of Proposition 3.1 can be reproduced without modification whenever the comparison principle of Assumption $(\mathbf{F})$ holds for $\mathcal{D}=[0, T] \times \mathcal{O}$. Moreover, the proof of Theorem 3.3 can be generalized to this context under the following additional assumption: there exist $\delta_{\mathcal{O}}>0$ and $\psi_{\mathcal{O}} \in C_{b}^{\infty}\left(\mathbb{R}^{d} \times \mathbb{R}^{d} ; \mathbb{R}_{+}\right)$such that, for all $x \in \mathbb{R}^{d}$,

(i) $x+\delta x^{\prime} \in \mathcal{O}$ for all $\delta \in\left(0, \delta_{\mathcal{O}}\right)$ and $x^{\prime} \in \mathbb{R}^{d}$ such that $\psi_{\mathcal{O}}\left(x, x^{\prime}\right) \neq 0$,

(ii) $\int_{\mathbb{R}^{d}} \psi_{\mathcal{O}}\left(x, x^{\prime}\right) d x^{\prime}=1$,

(iii) the support of $\psi_{\mathcal{O}}(x, \cdot)$ is contained in $[-1,1]^{d}$.

Indeed, it then suffices to replace the mollifier $\psi_{\delta}$ introduced in Step 2 of the proof of Theorem 3.3 by

$$
\psi_{\delta}\left(t^{\prime}, x^{\prime} ; x\right):=\delta^{-1-d} \phi\left(t^{\prime} / \delta\right) \psi_{\mathcal{O}}\left(x, x^{\prime} / \delta\right), \quad\left(t^{\prime}, x^{\prime}, x\right) \in \mathbb{R} \times \mathbb{R}^{d} \times \mathbb{R}^{d},
$$

for some smooth function $\phi \geq 0$ with support $[-1,0]$ such that $\int_{\mathbb{R}} \phi(t) d t=1$, and to define the convolution operation by

$$
\begin{aligned}
w \star \psi_{\delta}(t, x) & :=\int_{\mathbb{R}_{\mathbb{R}^{d}}} w\left(t^{\prime}, x^{\prime}\right) \psi_{\delta}\left(t^{\prime}-t, x^{\prime}-x ; x\right) d t^{\prime} d x^{\prime} \\
& =\int_{\mathbb{R} \times \mathbb{R}^{d}} w\left(t+t^{\prime}, x+x^{\prime}\right) \psi_{\delta}\left(t^{\prime}, x^{\prime} ; x\right) d t^{\prime} d x^{\prime} .
\end{aligned}
$$

For $\delta \leq \delta_{\mathcal{O}}$, the condition (i) above ensures that $x^{\prime}=x+\delta\left(x-x^{\prime}\right) / \delta \in \mathcal{O}$ whenever $x \in \mathcal{O}$ and $\psi_{\delta}\left(t^{\prime}-t, x^{\prime}-x ; x\right) \neq 0$. 
Similarly, the proofs of Theorems 2.3 and 2.5 do not change if we replace $[0, T] \times$ $\mathbb{R}^{d}$ by $[0, T] \times \mathcal{O}$. Under the additional assumption introduced above, Lemma 2.6 also extends to this case, and so do the proofs of Theorems 2.7 and 2.8 whenever the comparison principle of Assumption (D) holds for $\mathcal{D}=[0, T] \times \mathcal{O}$.

\section{Super-hedging under model uncertainty}

In this section, we apply the above results to a super-hedging problem under model uncertainty, where the dynamics of the underlying stocks and the interest rates depend on a process $\alpha$ which is only known to take values in the given set $A$. We consider an investor who wants to hedge an option written on some underlying stocks whose log-prices evolve according to

$$
X_{t, x}^{\alpha}=x+\int_{t} \mu\left(s, X_{t, x}^{\alpha}(s), \alpha_{s}\right) d s+\int_{t}^{\cdot} \sigma\left(s, X_{t, x}^{\alpha}(s), \alpha_{s}\right) d W_{s},
$$

for some unknown process $\alpha \in \mathcal{A}^{t}$. Thus, there can be uncertainty both about the drift and the volatility. Moreover, the range of possible coefficients may be statedependent. The investor also has a money market account at his disposal; however, the interest rates $\left(r^{b}\left(s, X_{t, x}^{\alpha}(s), \alpha_{s}\right)\right)_{s \leq T}$ and $\left(r^{l}\left(s, X_{t, x}^{\alpha}(s), \alpha_{s}\right)\right)_{s \leq T}$ for borrowing and lending are different and also depend on the process $\alpha$.

A trading policy for the investor is a process $\nu \in \mathcal{U}^{t}$ taking values in $U=\mathbb{R}^{d}$. Recall that $\mathcal{U}^{t}$ consists of processes which are in $L^{p}(\mathbb{P} \times d t)$ for some $p \geq 2$. In this section, we choose $p>2$. Each component $\nu^{i}$ corresponds to the monetary amount invested in the $i$-th stock. Given some initial capital $y \in \mathbb{R}$ at time $t$, the wealth process $Y_{t, x, y}^{\nu, \alpha}$ then evolves according to

$$
\begin{aligned}
Y_{t, x, y}^{\nu, \alpha}= & y+\int_{t}^{\cdot} \nu_{s}^{\top}\left\{d X_{t, x}^{\alpha}(s)+\frac{1}{2} \gamma\left(s, X_{t, x}^{\alpha}(s), \alpha_{s}\right) d s\right\} \\
& +\int_{t} \rho\left(s, X_{t, x}^{\alpha}(s), Y_{t, x, y}^{\nu, \alpha}(s), \nu_{s}, \alpha_{s}\right) d s \\
= & y+\int_{t}\left(\nu_{s}^{\top}\left\{\mu+\frac{1}{2} \gamma\right\}+\rho\right)\left(s, X_{t, x}^{\alpha}(s), Y_{t, x, y}^{\nu, \alpha}(s), \nu_{s}, \alpha_{s}\right) d s \\
& +\int_{t}^{\cdot} \nu_{s}^{\top} \sigma\left(s, X_{t, x}^{\alpha}(s), \alpha_{s}\right) d W_{s},
\end{aligned}
$$

where $\gamma$ is the vector containing the elements of the diagonal of the matrix $\sigma \sigma^{\top}$ and, with $1:=(1, \ldots, 1)^{\top}$,

$$
\rho(t, x, y, u, a):=\left[y-u^{\top} \mathbf{1}\right]^{+} r^{l}(t, x, a)-\left[y-u^{\top} \mathbf{1}\right]^{-} r^{b}(t, x, a)
$$

for $(t, x, y, u, a) \in[0, T] \times \mathbb{R}^{d} \times \mathbb{R} \times \mathbb{R} \times A$.

The process $\alpha$ may be interpreted as a directly observable risk factor such as the federal funds rate or an indicator for the state of the economy. In this case it clearly makes sense that the investor's actions may depend on $\alpha$ in a nonanticipative way. Alternatively, as the stocks $X_{t, x}^{\alpha}$ are observable, so are the volatilities $\sigma\left(s, X_{t, x}^{\alpha}(s), \alpha_{s}\right)$. Therefore, if $a \mapsto \sigma(s, x, a)$ is invertible, the process $\alpha$ is automatically observable. We thus allow the investor to choose a non-anticipative strategy $\mathfrak{u} \in \mathfrak{U}^{t}$. As a result, the super-hedging price is defined by

$$
v(t, x):=\inf \left\{y \in \mathbb{R}: \exists \mathfrak{u} \in \mathfrak{U}^{t} \text { s.t. } Y_{t, x, y}^{\mathfrak{u}, \alpha}(T) \geq g\left(X_{t, x}^{\alpha}(T)\right) \text { a.s. } \forall \alpha \in \mathcal{A}^{t}\right\}
$$


or the smallest price which allows to super-hedge for all $\alpha \in \mathcal{A}^{t}$.

We henceforth assume that $g$ is bounded and Lipschitz continuous, that $\mu$ and $\sigma$ are continuous functions, that

$(t, x, a) \in[0, T] \times \mathbb{R}^{d} \times A \mapsto\left(\mu, \sigma, r^{l}, r^{b}\right)(t, x, a)$ is continuous, bounded,

continuous in $(t, x)$ and Lipschitz continuous in $x$, both uniformly in $a$, and that $\sigma(t, x, a)$ is invertible for all $(t, x, a) \in[0, T] \times \mathbb{R}^{d} \times A$.

We also assume that

$$
r^{b} \geq r^{l}
$$

and that

$$
\begin{gathered}
\lambda^{b}:=\sigma^{-1}\left(\mu+\frac{1}{2} \gamma-r^{b}\right) \quad \text { and } \quad \lambda^{l}:=\sigma^{-1}\left(\mu+\frac{1}{2} \gamma-r^{l}\right) \text { are bounded, } \\
(t, x, a) \in[0, T] \times \mathbb{R}^{d} \times A \mapsto\left(\lambda^{b}, \lambda^{l}\right)(t, x, a) \text { does not depend on } x .
\end{gathered}
$$

We observe that the assumption (4.2) is in fact a no-arbitrage condition. In this setting, we have the following characterization of the super-hedging price.

Corollary 4.1. The function $v$ is continuous and the unique bounded viscosity solution of

$$
\begin{aligned}
& \inf _{a \in A}\left[\rho(\cdot, \varphi, D \varphi, a)+\frac{1}{2} \gamma(\cdot, a) D \varphi-\mathcal{L}^{a} \varphi\right]=0 \quad \text { on } \quad[0, T) \times \mathbb{R}^{d} \\
& \varphi(T, \cdot)=g \quad \text { on } \quad \mathbb{R}^{d},
\end{aligned}
$$

where

$$
\mathcal{L}^{a} \varphi(t, x):=\partial_{t} \varphi(t, x)+\frac{1}{2} \operatorname{Tr}\left[\sigma \sigma^{\top}(t, x, a) D^{2} \varphi(t, x)\right] .
$$

Proof. The present model satisfies the conditions (2.2) and (2.3) of Section 2 . Moreover, Assumption (A) holds with

$$
\hat{u}(t, x, y, z, a)=\left(\sigma^{-1}\right)^{\top}(t, x, a) z .
$$

To verify Assumption (B) consider $a \in A$ and $\mathfrak{u} \in \mathfrak{U}$. Then Itô's formula, our choice $p>2$ and (4.2) imply that the process $\left(e^{-\int_{t} r^{b}(s, a) d s} Y_{t, x, y}^{\mathfrak{u}, a}\right)$ is a super-martingale under the probability measure $\mathbb{Q}_{t, x}^{a}$ defined via the Doléans-Dade exponential

$$
\frac{d \mathbb{Q}_{t, x}^{a}}{d \mathbb{P}}=\mathcal{E}_{T}\left(-\int_{t} \lambda^{b}\left(s, X_{t, x}^{a}(s), a\right) d W_{s}\right) .
$$

In particular, if $\mathcal{D}_{<T} \times \mathbb{R} \ni\left(t_{n}, x_{n}, y_{n}\right) \rightarrow(T, x, y) \in \mathcal{D}_{T} \times \mathbb{R}$ and $\left(\mathfrak{u}_{n}\right)_{n \geq 1}$ are such that $\mathfrak{u}_{n} \in \mathfrak{U}^{t_{n}}$ and $Y_{t_{n}, x_{n}, y_{n}}^{\mathfrak{u}_{n}, a}(T) \geq g\left(X_{t_{n}, x_{n}}^{a}(T)\right)$ a.s., then $y_{n} \geq \mathbb{E}_{\mathbb{Q}_{t_{n}, x_{n}}^{a}}\left[g\left(X_{t_{n}, x_{n}}^{a}(T)\right)\right]$ for all $n \geq 1$, while (4.1) and the continuity of the bounded function $g$ ensure that $\mathbb{E}_{\mathbb{Q}_{t_{n}, x_{n}}^{a}}\left[g\left(X_{t_{n}, x_{n}}^{a}(T)\right)\right] \rightarrow g(x)$ as $n \rightarrow \infty$. Since $y_{n} \rightarrow y$, this shows that $y \geq g(x)$, so Assumption (B) holds.

As $g$ is bounded, the same super-martingale property implies that $v$ is bounded from below. Moreover, as $r^{l}$ is bounded, $v$ is also bounded from above, so we have (2.6). To verify Assumption (C), we use the definitions of $\hat{u}$ and $\rho$ to obtain that

$\mu_{Y}^{\hat{u}}(t, x, y, z, a):=\rho(t, x, y, \hat{u}(t, x, y, z, a), a)+\hat{u}(t, x, y, z, a)^{\top}\left\{\mu(t, x, a)+\frac{1}{2} \gamma(t, x, a)\right\}$ 
satisfies

$$
\left|\mu_{Y}^{\hat{u}}(t, x, y, z, a)\right| \leq|y|\left(\left|r^{b}\right| \vee\left|r^{l}\right|\right)(t, x, a)+|z|\left(\left|\lambda^{l}\right| \vee\left|\lambda^{b}\right|\right)(t, x, a)
$$

and

$$
\begin{aligned}
\left|\mu_{Y}^{\hat{u}}(t, x, y, z, a)-\mu_{Y}^{\hat{u}}\left(t, x, y^{\prime}, z^{\prime}, a\right)\right| \leq & \left|y-y^{\prime}\right|\left(\left|r^{b}\right| \vee\left|r^{l}\right|\right)(t, x, a) \\
& +\left|z-z^{\prime}\right|\left(\left|\lambda^{b}\right| \vee\left|\lambda^{l}\right|\right)(t, x, a)
\end{aligned}
$$

for all $(t, x, a) \in[0, T] \times \mathbb{R}^{d} \times A$ and $(y, z),\left(y^{\prime}, z^{\prime}\right) \in \mathbb{R} \times \mathbb{R}^{d}$. The Lipschitz continuity in the $x$-variable follows immediately from (4.3). Assumption $(\mathbf{E})$ is satisfied 3 due to $r^{l} \leq r^{b}$. Finally, Assumption (D) follows from standard arguments under our Lipschitz continuity conditions; cf. [9].

\section{References}

[1] M. Avellaneda, A. Levy, and A. Parás. Pricing and hedging derivative securities in markets with uncertain volatilities. Appl. Math. Finance, 2(2):73-88, 995.

[2] G. Barles and E. R. Jakobsen. On the convergence rate of approximation schemes for Hamilton-Jacobi-Bellman equations. M2AN Math. Model. Numer. Anal., 36(1):33-54, 2002.

[3] G. Barles and P. E. Souganidis. Convergence of approximation schemes for fully nonlinear second order equations. Asymptotic Anal., 4(3):271-283, 991.

[4] E. Bayraktar and J. Li. Stochastic perron for stochastic target games. arXiv preprint arXiv:1408.6799, 2014.

[5] B. Bouchard, R. Elie, and N. Touzi. Stochastic target problems with controlled loss. SIAM J. Control Optim., 48(5):3123-3150, 2009.

[6] B. Bouchard, L. Moreau, and M. Nutz. Stochastic target games with controlled loss. To appear in Ann. Appl. Probab., 2012.

[7] B. Bouchard and M. Nutz. Arbitrage and duality in nondominated discretetime models. To appear in Ann. Appl. Probab., 2013.

[8] R. Buckdahn and J. Li. Stochastic differential games and viscosity solutions of Hamilton-Jacobi-Bellman-Isaacs equations. SIAM J. Control Optim., 47(1):444-475, 2008.

[9] M. G. Crandall, H. Ishii, and P. L. Lions. User's guide to viscosity solutions of second order partial differential equations. Bull. Amer. Math. Soc., 27(1):1-67, 1992.

[10] C. Dellacherie and P. A. Meyer. Probabilities and Potential B. North Holland, Amsterdam, 1982.

[11] L. Denis and C. Martini. A theoretical framework for the pricing of contingent claims in the presence of model uncertainty. Ann. Appl. Probab., 16(2):827$852,2006$.

\footnotetext{
${ }^{3}$ We may observe here that this concavity condition is satisfied in a very natural way in the context of mathematical finance, where wealth dynamics are typically either linear or exhibit concave nonlinearities. The concavity is related to the absence of arbitrage opportunities; in the present example, the fact that $r^{b} \geq r^{l}$.
} 
[12] W. H. Fleming and P. E. Souganidis. On the existence of value functions of two-player, zero-sum stochastic differential games. Indiana Univ. Math. J., 38(2):293-314, 1989.

[13] H. Ishii. On the equivalence of two notions of weak solutions, viscosity solutions and distribution solutions. Funkcial. Ekvac, 38(1):101-120, 1995.

[14] R. Jensen. The maximum principle for viscosity solutions of fully nonlinear second order partial differential equations. Arch. Rational Mech. Anal., 101(1):1-27, 1988.

[15] N. V. Krylov. On the rate of convergence of finite-difference approximations for Bellman's equations with variable coefficients. Probab. Theory Related Fields, 117(1):1-16, 2000.

[16] A. Neufeld and M. Nutz. Superreplication under volatility uncertainty for measurable claims. Electron. J. Probab., 18(48):1-14, 2013.

[17] J. Neveu. Discrete-Parameter Martingales. North-Holland, Amsterdam, 1975.

[18] E. Pardoux. Backward stochastic differential equations and viscosity solutions of systems of semilinear parabolic and elliptic pdes of second order. Progr. Probab., 42:79-128, 1998.

[19] S. Peng. A generalized dynamic programming principle and Hamilton- JacobiBellman equation. Stochastics Stochastics Rep., 38(2):119-134, 1992.

[20] S. Peng. Backward stochastic differential equations and applications to optimal control. Appl. Math. Optim., 27(2):125-144, 1993.

[21] S Peng. Bsde and stochastic optimizations. Topics in Stochastic Analysis, 1997.

[22] S. Peng. Nonlinear expectations and stochastic calculus under uncertainty. arXiv preprint arXiv:1002.4546v1, 2010.

[23] T. Pham and J. Zhang. Two person zero-sum game in weak formulation and path dependent Bellman-Isaacs equation. arXiv preprint arXiv:1209.6605v1, 2012.

[24] M. Sirbu. Stochastic Perron's method and elementary strategies for zero-sum differential games. SIAM J. Control Optim., 52(3):1693-1711, 2014.

[25] H. M. Soner and N. Touzi. Dynamic programming for stochastic target problems and geometric flows. J. Eur. Math. Soc. (JEMS), 4(3):201-236, 2002.

[26] H. M. Soner and N. Touzi. Stochastic target problems, dynamic programming, and viscosity solutions. SIAM J. Control Optim., 41(2):404-424, 2002.

[27] H. M. Soner, N. Touzi, and J. Zhang. Martingale representation theorem for the G-expectation. Stochastic Process. Appl., 121(2):265-287, 2011.

[28] H. M. Soner, N. Touzi, and J. Zhang. Wellposedness of second order backward SDEs. Probab. Theory Related Fields, 153(1-2):149-190, 2012.

[29] H. M. Soner, N. Touzi, and J. Zhang. Dual formulation of second order target problems. Ann. Appl. Probab., 23(1):308-347, 2013.

[30] D. Talay and Z. Zheng. Worst case model risk management. Finance Stoch., 6(4):517-537, 2002. 
[31] R. Tevzadze, T. Toronjadze, and T. Uzunashvili. Robust utility maximization for a diffusion market model with misspecified coefficients. Finance Stoch., 17(3):535-563, 2013. 